\title{
Feedforward and feedback projections of caudal belt and parabelt areas of auditory cortex: refining the hierarchical model
}

\author{
Troy A. Hackett ${ }^{1 *}$, Lisa A. de la Mothe ${ }^{2}$, Corrie R. Camalier ${ }^{1,3}$, Arnaud Falchier ${ }^{4,5}$, Peter Lakatos ${ }^{4,5}$, \\ Yoshinao Kajikawa ${ }^{4,5}$ and Charles E. Schroeder ${ }^{4,5}$
}

${ }^{1}$ Department of Hearing and Speech Sciences, Vanderbilt University School of Medicine, Nashville, TN, USA

${ }^{2}$ Department of Psychology, Tennessee State University, Nashville, TN, USA

${ }^{3}$ Laboratory of Neuropsychology, National Institutes of Mental Health, Bethesda, MD, USA

${ }^{4}$ Cognitive Neuroscience and Schizophrenia Program, Nathan Kline Institute, Orangeburg, NY, USA

${ }^{5}$ Department of Psychiatry, Columbia University College of Physicians and Surgeons, New York, NY, USA

\section{Edited by:}

Yukiko Kikuchi, Newcastle University Medical School, UK

\section{Reviewed by:}

Lizabeth M. Romanski, University of Rochester School of Medicine and Dentistry, USA

Brian H. Scott, National Institute of Mental Health, USA

\section{*Correspondence:}

Troy A. Hackett, Department of Hearing and Speech Sciences, Vanderbilt University School of Medicine, 465 21st Avenue South MRB-3 Suite 7110, Nashville,

TN 37232, USA

e-mail: troy.a.hackett@vanderbilt.edu
Our working model of the primate auditory cortex recognizes three major regions (core, belt, parabelt), subdivided into thirteen areas. The connections between areas are topographically ordered in a manner consistent with information flow along two major anatomical axes: core-belt-parabelt and caudal-rostral. Remarkably, most of the connections supporting this model were revealed using retrograde tracing techniques. Little is known about laminar circuitry, as anterograde tracing of axon terminations has rarely been used. The purpose of the present study was to examine the laminar projections of three areas of auditory cortex, pursuant to analysis of all areas. The selected areas were: middle lateral belt (ML); caudomedial belt (CM); and caudal parabelt (CPB). Injections of anterograde tracers yielded data consistent with major features of our model, and also new findings that compel modifications. Results supporting the model were: (1) feedforward projection from $\mathrm{ML}$ and $\mathrm{CM}$ terminated in CPB; (2) feedforward projections from $\mathrm{ML}$ and CPB terminated in rostral areas of the belt and parabelt; and (3) feedback projections typified inputs to the core region from belt and parabelt. At odds with the model was the convergence of feedforward inputs into rostral medial belt from $\mathrm{ML}$ and CPB. This was unexpected since CPB is at a higher stage of the processing hierarchy, with mainly feedback projections to all other belt areas. Lastly, extending the model, feedforward projections from CM, ML, and CPB overlapped in the temporal parietal occipital area (TPO) in the superior temporal sulcus, indicating significant auditory influence on sensory processing in this region. The combined results refine our working model and highlight the need to complete studies of the laminar inputs to all areas of auditory cortex. Their documentation is essential for developing informed hypotheses about the neurophysiological influences of inputs to each layer and area.

Keywords: connections, brain, monkey, functional organization, laminar, architecture, anatomy, laminar

\begin{abstract}
Abbreviations: A1, Auditory area 1 (core); AChE, Acetylcholinesterase; AL, Anterolateral area (belt); AS, Arcuate sulcus; Cis, Circular sulcus; CL, Caudolateral area (belt); CM, Caudomedial area (belt); CO, Cytochrome oxidase; $\mathrm{CPB}$, Caudal parabelt area (parabelt); CS, Central sulcus; Id, Insula, dysgranular; Ig, Insula, granular; IPS, Intraparietal sulcus; ITG, Inferior temporal gyrus; Lim, Limitans nucleus; LS, Lateral sulcus; LuS, Lunate sulcus; MGad, Medial geniculate complex, anterodorsal division; MGC, Medial geniculate complex; MGd, Medial geniculate complex, dorsal division; MGm, Medial geniculate complex, magnocellular division; MGpd, Medial geniculate complex, posterodorsal division; MGv, Medial geniculate complex, ventral division; ML, Middle lateral area (belt); MT, Middle temporal area; Pro, Proisocortical area; proA, Prokoniocortex area; PS, Principal sulcus; PV, Parvalbumin; R, Rostral area (core); Ri, Retroinsular area; RM, Rostromedial area (belt); RPB, Rostral parabelt area (parabelt); RT, Rostrotemporal area (core); RTL, Rostrotemporal lateral area (belt); RTM, Rostrotemporal medial area (belt); S2, Somatosensory area 2; Sg, Suprageniculate nucleus; STG, Supeior temporal gyrus; STS, Superior temporal sulcus; TPO, Temporal parietal occipital area; TPOc, TPO, caudal sector; TPOr, TPO, rostral sector; Tpt, Temporal parietotemporal area; VGluT1, Vesicular glutamate transporter 1; VGluT2, Vesicular glutamate transporter 2 .
\end{abstract}

\section{INTRODUCTION}

The auditory cortex of primates is spread out over a large portion of the superior temporal gyrus (STG) and plane. Current models recognize 13 areas, grouped into three major regions (core, belt, parabelt). The identification and classification of areas and regions is based on interpretation of their neuroanatomical and neurophysiological profiles (Pandya et al., 1969; Pandya and Sanides, 1973; Burton and Jones, 1976; Jones and Burton, 1976; Imig et al., 1977; Fitzpatrick and Imig, 1980; Galaburda and Pandya, 1983; Cipolloni and Pandya, 1989; Morel and Kaas, 1992; Morel et al., 1993; Jones et al., 1995; Kosaki et al., 1997; Hackett et al., 1998; de la Mothe et al., 2006; Smiley et al., 2007; Hackett and de la Mothe, 2009). Among the most informative and distinguishing features of the three regions are the topographic patterns of connectivity within and between them. The three areas that comprise the core region are densely interconnected 
with about eight areas in the surrounding belt region. The belt areas have strong connections with parabelt region, which is currently divided into two areas. The core areas have only sparse connections with the parabelt.

On the basis of these connections, a regional hierarchy has been proposed in which information received by the core is sequentially processed by areas in the belt, and then the parabelt (Hackett et al., 1998). A second gradient has also been proposed along the caudal-rostral axis of the temporal lobe, based on the patterns of connections and architectonic gradients (Hackett, 2011). Although scant data are available, the known laminar projections suggest that information tends to flow from caudal to rostral areas in a feedforward manner (dominant inputs to layer 4 ), whereas projections from rostral onto caudal areas tend to exhibit feedback laminar profiles (dominant inputs to supragranular and/or infragranular layers, especially layer 1) (Rockland and Pandya, 1979; Felleman and Van Essen, 1991). There is also a bit of evidence that the most caudal auditory belt areas (caudomedial, CM; caudolateral, CL) direct some feedforward projections caudally toward auditory-related areas in the temporoparietal junction, such as Tpt (Fitzpatrick and Imig, 1980; Galaburda and Pandya, 1983; de la Mothe et al., 2006). Thus, information flow within the auditory cortex appears to move along two major anatomical axes: core-belt-parabelt and caudal-rostral. Correlated with these anatomical patterns are gradients in neuronal response properties. Frequency tuning bandwidth, response latencies and stimulus specificity generally increase along these axes, whereas temporal precision tends to decrease (Rauschecker et al., 1995, 1997; Rauschecker, 1998a,b; Rauschecker and Tian, 2004; Lakatos et al., 2005a; Bendor and Wang, 2008; Petkov et al., 2008; Kusmierek and Rauschecker, 2009; Kikuchi et al., 2010; Scott et al., 2011; Camalier et al., 2012; Kusmierek et al., 2012).

Beyond the confines of the auditory cortex, information from the belt and parabelt areas reaches multiple auditory-related areas distributed throughout the brain. (Tranel et al., 1988; Kosmal et al., 1997; Hackett et al., 1999; Romanski et al., 1999a,b; Cavada et al., 2000; Lewis and Van Essen, 2000; Falchier et al., 2002, 2009; Ghashghaei and Barbas, 2002; Lavenex et al., 2002; Petrides and Pandya, 2002; Yukie, 2002; Rockland and Ojima, 2003; Barbas, 2007; Smiley et al., 2007; Saleem et al., 2008, 2013; Markov et al., 2014). A rostrally-directed stream reaches targets in the temporal pole, ventral, rostral and medial prefrontal cortex, rostral cingulate, parahippocampal areas and the amygdala. A caudallydirected stream flows from the caudal belt and parabelt areas into the temporoparietal junction, posterior parietal and occipital regions (such as secondary visual cortex), caudal and dorsal prefrontal areas, dorsal cingulate and parahippocampal areas. Additional output streams flow laterally from the belt and parabelt regions to the upper bank of the superior temporal sulcus (STS) and medially into the insula and retroinsular areas within the lateral sulcus (Galaburda and Pandya, 1983; Hackett et al., 1998; de la Mothe et al., 2006; Smiley et al., 2007).

At present, the wiring diagrams that inform our models of auditory cortical function are low in resolution. The most widely used schematics depict the auditory areas on surface maps of the brain, using lines and arrows to denote a connection between one area and another (Figures 1A,B,D). These diagrams are a useful guide for describing the basic layout and connections of the auditory cortex, but reveal nothing about the laminar distributions of the somata, dendrites and axon terminals that comprise those connections and contribute to their functional importance (e.g., feedforward, feedback, etc.).

Unfortunately, these kinds of low-resolution maps reflect most of the current knowledge base. There are two reasons for this. First, most of the intrinsic and extrinsic connections identified in the experiments cited above used retrograde tracers, which permit identification of the input sources (neuronal somata) to the area targeted by a tracer injection. Relatively few of these studies used tracers with anterograde transport properties, which reveal the laminar projections from an area to its targets. Second, several studies utilized flattened brain preparations (cut parallel to the pial surface) for areal reconstruction of the patterns of labeled cells. While these methods are advantageous for creating surface maps of the connections between areas and regions, information about the laminar circuitry is not preserved. Fortunately, a few studies have been published in which the connections were studied in coronal sections using at least some tracers with anterograde transport properties (Fitzpatrick and Imig, 1980; Galaburda and Pandya, 1983; Aitkin et al., 1988; de la Mothe et al., 2006; de la Mothe et al., 2012). As noted above, sufficient data could be gleaned from these studies to support hypotheses about information flow along the two major axes (Hackett, 2011). However, these data are far from complete, derived from mixed primate species using different methods, and inconsistent at several levels of analysis. Lacking, and desperately needed, is an extended series of detailed anatomical studies in which anterograde and retrograde tracers are employed to more completely work out the laminar distribution patterns that actually comprise the connections between areas. Ideally, a survey of the input/output connections of each area should be obtained so that a complete wiring diagram of the auditory cortex could be made available. Such maps were developed over two decades ago for primate visual cortex (Felleman and Van Essen, 1991), and studies of both visual and somatosensory cortex have since progressed toward defining the connections of morphologically and neurochemically distinct neuronal subpopulations in every layer and sublayer (Callaway, 2002; Thomson and Bannister, 2003; Bannister, 2005; Douglas and Martin, 2007; Briggs, 2010; Feldmeyer et al., 2013). This implies that for primate auditory cortex, we are at the early stages of obtaining the kinds of basic data that were summarized over 20 years ago by researchers studying the visual cortex. This is an essential task, and one that will require sustained effort to complete.

With that in mind, the present study represents the beginning of what we intend to expand into a comprehensive accounting of the intrinsic connections of the primate auditory cortex. By injecting tracers with bidirectional transport properties in caudal belt $(\mathrm{ML}, \mathrm{CM})$ and parabelt $(\mathrm{CPB})$ areas, our primary goals were to begin acquisition of the anatomical data, and at the same time test key predictions of our working model. For example, if the belt is driving activity in the parabelt, then projections from the belt should (at least) target layer 4 (L4) of 


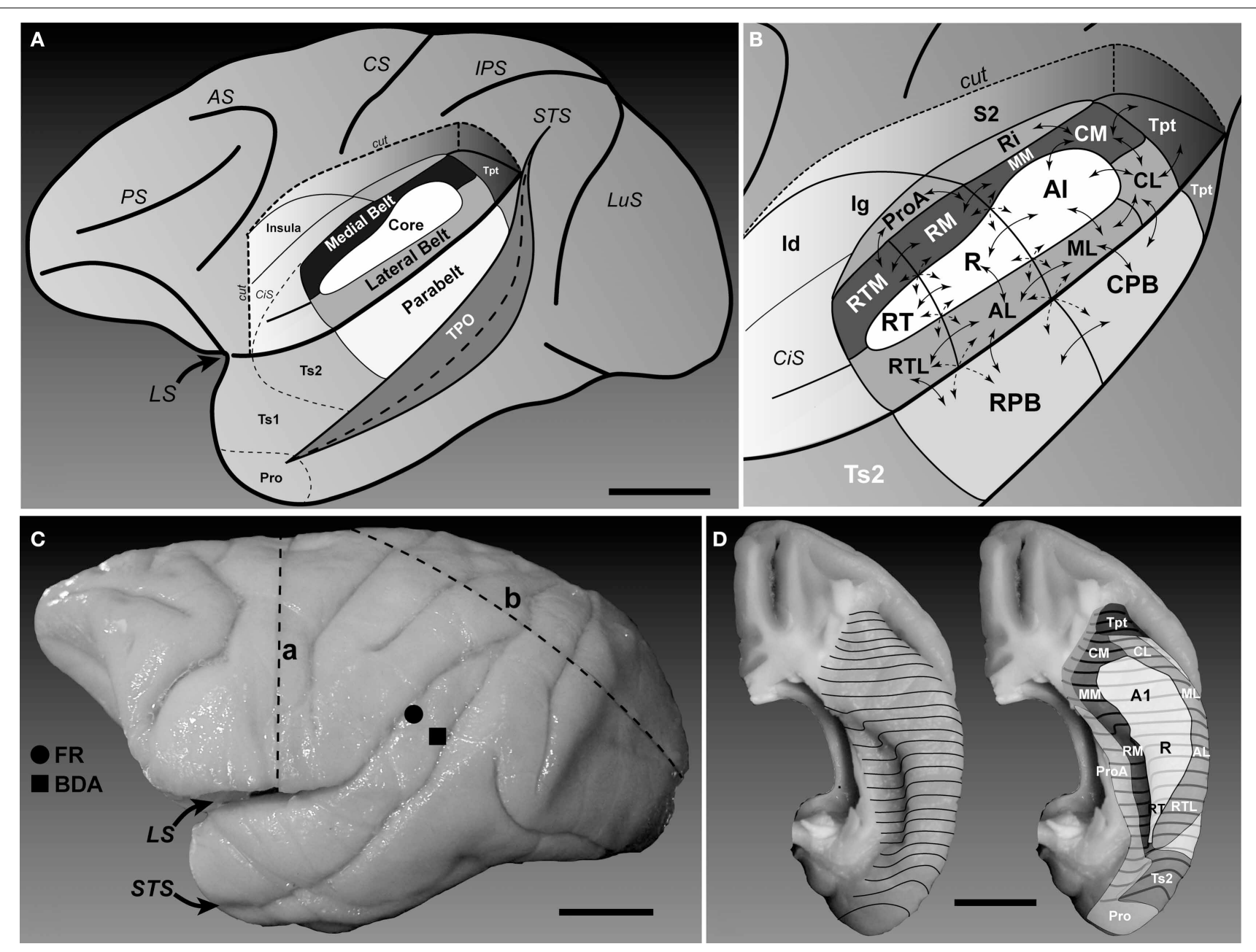

\section{FIGURE 1 | Location and subdivisions of the auditory cortex in} macaque monkeys. (A) Lateral view of the left hemisphere showing position of auditory and auditory-related regions in the superior temporal region. The parabelt region is located on the surface of the superior temporal gyrus (STG). The core and belt regions lie on the superior temporal plane, visible after graphical removal of the overlying parietal and frontal opercula (cut). Area TPO lies on the upper bank of the superior temporal sulcus. The locations of the areas on the STG rostral to auditory cortex (Ts2, Ts1, and Pro) are also labeled (after Galaburda and Pandya, 1983). (B) Location and connections of areas within and around auditory cortex (see list of abbreviations for details). Core region: A1, R, RT; Medial belt region: $C M, M M, R M, R T M$; Lateral belt region: $C L, M L, A L, R T L$; Parabelt region: CPB, RPB; auditory-related fields, Tpt, Ri, Pro, S2, Ig, Id, Ts2. Arrows denote simplified patterns of connections between areas. Connections between non-adjacent areas not shown. (C) Photograph of the left hemisphere of Case 2 showing locations of injections (FR, filled circle; BDA, filled square) and blocking cuts (dashed lines a and b).

Microtome sections were cut parallel to line b. (D) Dorsal views of the superior temporal plane showing locations of core, belt, and surrounding regions. The approximate locations of several areas are labeled. Caudal is up, lateral is right. Black contour lines run across the brain surface from medial (left) to lateral (right). Scale bars, $10 \mathrm{~mm}$. the parabelt. Similarly, if caudal areas are driving rostral areas, then projections from the caudal areas should target L4 of the rostral areas. In addition, these experiments provided us with an opportunity to better characterize the nature of the inputs to the temporoparietal occipital area (TPO) on the upper bank of the STS, which is broadly connected with numerous auditory, visual, somatosensory, prefrontal, and posterior parietal areas. Earlier observations suggested that projections from the STG project to L4 and other layers of TPO (Cusick et al., 1995; Seltzer et al., 1996), indicating that the parabelt could be the main source of feedforward auditory input to this multisensory region.

\section{MATERIALS AND METHODS}

Three macaque monkeys were used in this study (1 macaca mulatta, 2 macaca radiata). All procedures involving animals were conducted in accordance with international standards on animal welfare, followed NIH Guidelines for the Care and Use of Laboratory Animals, and were approved in advance by the Vanderbilt University Institutional Animal Care and Use Committee.

\section{GENERAL SURGICAL PROCEDURES}

Aseptic techniques were employed during all surgical procedures. Animals were premedicated with cefazolin $(25 \mathrm{mg} / \mathrm{kg})$, 
dexamethasone $(2 \mathrm{mg} / \mathrm{kg})$, and robinul $(0.015 \mathrm{mg} / \mathrm{kg})$. Anesthesia was induced by intramuscular injection of ketamine hydrochloride $(10 \mathrm{mg} / \mathrm{kg})$ then maintained by continuous isoflurane (2-3\%) inhalation blended with $100 \%$ oxygen $(1 \mathrm{~L} / \mathrm{min})$ through an endotracheal tube. Body temperature was held at $37^{\circ} \mathrm{C}$ with a water circulating heating pad. Heart rate, expiratory $\mathrm{CO}_{2}$, and $\mathrm{O}_{2}$ saturation were continuously monitored throughout the surgery and used to adjust anesthetic depth. For all surgical procedures, the head was held by hollow ear bars affixed to a stereotaxic frame (David Kopf Instruments, Tujunga, CA).

In Cases 1 and 3, injections of ML and CM were made using a vertical approach through chronic recording chambers (Crist Instruments, Hagerstown, MD) implanted over the auditory cortex in the left hemisphere. Injections were made through the injection ports of 24-channel linear array electrodes (U-Probe, Plexon Inc., Dallas, Tx) after completion of chronic electrophysiological recordings. The details of this procedure are explained in Smiley et al. (2007), where we first used the U-Probe for this purpose. Briefly, current source density analysis was used to initially position the deepest electrode channels between the pia and white matter. Slight adjustments in position are made to center the injection port at the prominent current sink in L4. The full volume of tracer (Table 1) was injected in 3 equal boluses, which disperses by capillary action up and down the electrode shaft, effectively depositing tracer across all cortical layers.

In Case 2, a midline incision was made exposing the skull, followed by retraction of the temporal muscle. A craniotomy was performed exposing the left dorsal STG, lateral fissure, and overlying parietal cortex. After retraction of the dura, warm sterilized silicone oil was applied to the brain to prevent desiccation of the cortex. Tracer injections were made into target areas through a pulled glass pipette affixed to a $1 \mu \mathrm{l}$ Hamilton syringe. The pipette was advanced into cortex under stereo microscopic observation to a depth of $1000 \mu \mathrm{m}$ using a stereotaxic micromanipulator. After manual pressure injection of tracer into each target area (Table 1), the syringe was held in place for $10 \mathrm{~min}$ under continuous observation to maximize uptake and minimize leakage. Injection the CPB were made directly into the lateral surface of the STG after removal of the dura. Injection of ML was achieved by slight retraction of the banks of the lateral fissure, as previously described (Hackett et al., 2005).

\section{TRACER INJECTIONS}

In all cases, tracers were injected by pressure into target areas of the auditory cortex. Table 1 contains the relevant experimental details of each case, including tracer type, tracer volume, injection device, and area injected. In Cases 1 and 3, tracer injections were made subsequent to a series of electrophysiological recordings to more accurately identify the target area and surrounding areas. In Case 1, the injection into area ML was made through the recording chamber during a routine awake-behaving recording session. Recordings in this case broadly covered 10 areas of auditory cortex, as reported in Camalier et al. (2012). In Case 3, the injection into the rostral-medial limb of area CM was made through an established recording chamber following recordings focused on areas A1 and CM. Spatial mapping density was not dense in either of these cases, and did not significantly interfere with tracer transport or architectonic assays. In some figures, electrode tracks or lesions can be seen and these are marked with asterisks. In Case 2, the injections into areas ML (near its the caudal and lateral borders) and CPB (caudal and ventral quadrant) were made directly into cortex through a craniotomy under general anesthesia (see above) and in the absence of electrophysiology. Stereotaxic coordinates and surface landmarks were used to identify the target locations. In an attempt to avoid involving the dorsal $\mathrm{CPB}$, the ML injection was made medial to the middle cerebral vein, by slight retraction of the dorsal bank of the lateral sulcus (Hackett et al., 2005). Note that the proximity of the injection to the CPB border (Figure 4E). The spread of tracer was minimal, but may have encroached slightly into the $\mathrm{CPB}$, and if so, mainly in L13a. Although the injection obscured architectonic features and absolute confirmation, the dense retrograde labeling in A1 is consistent with a significant deposit in $\mathrm{ML}$, since $\mathrm{CPB}$ injections rarely produce labeled cells in A1 (Supplementary Figure 2 and Supplementary Table 2). If the FR tracer was transported by any cells in the dorsal CPB, we are unable to determine that from the labeling patterns observed.

Three tracers were used in these studies: cholera toxin subunit B (CTB) (Vector Labs); $10 \mathrm{kDa}$ tetramethylrhodamine (aka fluororuby, abbreviated as FR) (Molecular Probes); $10 \mathrm{kDa}$ biotinylated dextran amine (BDA) (Sigma). FR and BDA have the potential for bidirectional axonal transport (anterograde and retrograde), but are most sensitive as anterograde tracers and produce well-defined labeling of axon arbors and terminal puncta. CTB is a very sensitive retrograde tracer, but uptake often produces strong anterograde transport, as in Case 1 and our previous studies in marmosets (de la Mothe et al., 2006). Rather than punctate labeling of terminals and their axonal arbors, anterograde transport of CTB typically produces a "dust-like" deposit in the terminal zone intermingled with some punctate terminal labeling.

\begin{tabular}{|c|c|c|c|c|c|c|c|}
\hline \multirow[t]{2}{*}{2} & M. radiata & $\mathrm{F}$ & $\mathrm{ML}$ & Hamilton & 10 kDa FR & 10 & 0.8 \\
\hline & & & $\mathrm{CPB}$ & & 10 kDa BDA & 10 & $0.5 \times 2$ \\
\hline 3 & M. mulatta & $\mathrm{F}$ & Rostral CM & U-Probe & 10 kDA BDA & 10 & 0.4 \\
\hline
\end{tabular}

Areas of tracer injections (ML, middle lateral belt; CM, caudal medial belt; CPB, caudal parabelt). Neuroanatomical tracers (CTB, cholera toxin subunit B; BDA, biotinylated dextran amine; FR, fluororuby). Aqueous concentrations and volumes injected are listed for each tracer. 
This labeling is fine enough to be localized to specific to laminae and sublaminae, but the contacts of individual terminal puncta cannot usually be resolved.

\section{PERFUSION AND HISTOLOGY}

After a 14-21 days survival period, a lethal dose of pentobarbital $(120 \mathrm{mg} / \mathrm{kg})$ was administered intravenously. Just after cardiac arrest the animal was perfused through the heart with cold $\left(4^{\circ} \mathrm{C}\right)$ saline, followed by cold $\left(4^{\circ} \mathrm{C}\right) 4 \%$ paraformaldehyde dissolved in $0.1 \mathrm{M}$ phosphate buffer. Following perfusion the brains were removed and photographed. The cerebral hemispheres were blocked and placed in 30\% sucrose for 3 days. The cerebral hemispheres of each case were cut at slightly different angles. In Cases 1 and 3 , the angle was very close to coronal, matching the angle of electrode penetrations through the recording chamber. In case 2, the angle was perpendicular to the lateral sulcus in the caudal to rostral direction at $40 \mu \mathrm{m}$, as shown in Figure 1C (line " $\mathrm{b}$ "). This minimized cross-cutting across cortical columns for areas in the lateral, superior temporal, inferior parietal, and central sulci.

In each brain, series of 12 sections were alternately processed for the following set of histochemical markers: (i) fluorescent tracer microscopy; (ii) biotinylated dextran amine (BDA) or cholera toxin subunit B (CTB); (iii) acetylcholinesterase (AChE) (Geneser-Jensen and Blackstad, 1971); (iv) stained for Nissl substance with thionin. Additional reactions were performed in some cases to facilitate reconstruction, or obtain data for other studies: (v) cytochrome oxidase (Wong-Riley, 1979); (vi) parvalbumin (PV); (vii) vesicular glutamate transporters 1 (VGluT1) and 2 (VGluT2) (Hackett and de la Mothe, 2009); (ix) neuron specific nuclear protein, NeuN (Hackett and de la Mothe, 2009), and (x) myelinated fibers (MF) (Gallyas, 1979). In cases 2 and 3, multifluorescent immunohistochemistry (IHC) of NeuN and VGluT2, VGluT1 or PV were combined with fluorescent detection of tracers in single sections to relate areal and laminar boundaries to the locations of axon terminals and somata. Auditory areas were identified using these markers according to detailed architectonic criteria established in previous studies of macaque monkeys (Hackett et al., 2001; Smiley et al., 2007; Hackett and de la Mothe, 2009), and also applied to marmoset monkeys (de la Mothe et al., 2006). In brief, the cytoarchitecture of the core areas stands out for a broad densely packed L4, separated from L6 by a cell-sparse L5. The middle layers of the core stain darkly for PV, VGluT2, MF, and AChE compared to sharp reductions in density at borders with adjacent belt areas. The parabelt transition from lateral belt to parabelt is usually within $1 \mathrm{~mm}$ of the edge of the lateral sulcus. It is not always sharply demarcated, but characterized by a reduction in L4 density of these markers. There is a gradual reduction in the prominence of these markers from along the caudal-to-rostral axis, which is used to distinguish adjacent areas along that axis. The border between parabelt and TPO is generally near the lateral edge of the STS, and its precise location also appears to meander somewhat along the rostral-caudal axis. Supplementary Figures 1A,B shows an example of fluorescent NeuN IHC paired with an adjacent section stained for VGluT2 and identification of areas in a coronal section through A1. The panels below illustrate how laminar boundaries were located in sections containing FR and BDA tracers in three different areas $(\mathrm{C}-\mathrm{F}$, area $\mathrm{ML} ; \mathrm{G}-\mathrm{J}$, area $\mathrm{MM}, \mathrm{K}-\mathrm{N}$, area TPO).

\section{MICROSCOPY AND RECONSTRUCTION OF SECTIONS}

Digital images of brightfield sections were acquired using a Neurolucida system (MicroBright Field, Inc., Williston, VT) and Nikon 80i microscope. Fluorescent images were acquired using a Hamamatsu Orca digital camera and Nikon 90i microscope. All of the images in Figures 2-9 are photomontages stitched from a matrix of multiple photographs obtained using a $10 \times$ objective and Nikon Elements AR software. These images were cropped, adjusted for brightness and contrast using Adobe Photoshop CS6 software. Images of sections containing transported tracers were selected at regular intervals $(\sim 1: 24)$ for illustration in rostral-to-caudal sequence. Final figures containing images and line drawings were made using Adobe Illustrator CS6 (Adobe Systems, Inc.). All descriptions of anterograde tracer deposits recorded in the text and figures were based on calculation of relative optical density in each layer of each cortical area. Digitized images of each cortical area (see Figures 5, 7, 9) were converted to 8-bit grayscale images and imported into ImageJ at full resolution (Rasband, W.S., ImageJ, U. S. National Institutes of Health, Bethesda, Maryland, USA, http://imagej.nih.gov/ij/, 1997-2014). For each cortical area, inverted grayscale levels (GL) from 0 (white) to 255 (black) were measured in each layer from regions of interest encompassing labeled terminals drawn using the polygon tool, avoiding artifacts, blood vessels, and retrogradely labeled cells. Background grayscale levels (BL) were measured from a separate region devoid of terminals in the white matter just below layer 6. The Gray Level Index (GLI) was calculated as follows: $\mathrm{GLI}=(\mathrm{GL}-\mathrm{BL}) / \mathrm{BL}$. These values are recorded in Supplementary Table 1, and used to set the intensity of layers in each panel of Figure 10. A "feedforward" (FF) connection type was defined as an axonal projection that produced concentrated terminal labeling in L4. Almost invariably, feedforward inputs to L4 of a recipient area were accompanied by band of labeled axons and terminals concentrated in other layers, most often L1 and L6. This was sometimes accompanied by weaker axon and terminal labeling in the intervening layers (L2-3B, L5). We refer to these projections as "lateral" connections. Projections that were concentrated in L1 or L1 and L6 are referred to as "feedback." These designations are based on prior studies (Rockland and Pandya, 1979; Felleman and Van Essen, 1991).

\section{RESULTS}

\section{CASE 1: ML INJECTION}

The sentinel case, which initiated the present study, was Case 1 (Figure 2). An injection of CTB was placed into ML, as part of an ongoing study of retrograde transport to the thalamus. Figure 2 contains images of tissue sections at the level of ML, containing the injection site $(C, D)$, and more rostral sections at the level of the RPB $(A, B)$. At each level, sections stained for CTB $(A, C)$ are depicted with a nearby section stained for AChE $(B, D)$, as an example of the architecture that was used to identify areas of auditory cortex. In the panels below (a-e), higher magnification images from the RPB (a), CPB (b), A1 (c), and TPO (d,e) show retrograde and anterograde transport across laminae. 


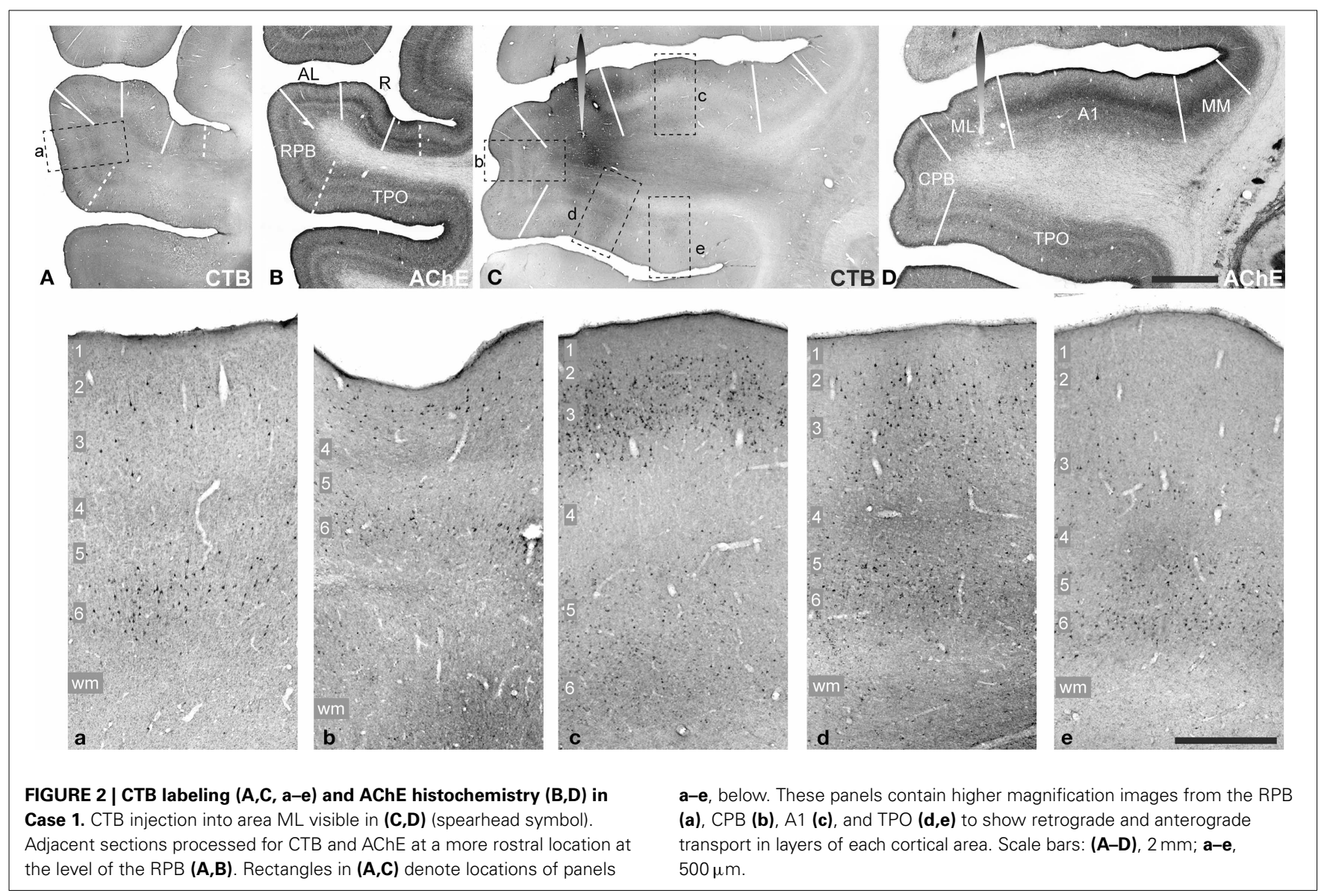

The injection spanned all cortical layers and also appeared to involve the white matter directly below layer 6 . Transport from this injection produced retrograde labeling of somata in nearly all areas of auditory cortex and ventrally across much of TPO on the upper bank of the STS. In addition, although CTB does not always produce detectable anterograde labeling, this case was an exception, as anterograde deposits were reliably present in areas where retrogradely labeled cells were found, and also in laminae (e.g., L1, L4) where they were not. Of particular interest was that anterograde transport was present in L4 and L6 of the RPB and CPB (Figures 2Aa, Cb), and extended across most of TPO (Figure 2Cd,e). Dense patches of overlapping anterograde and retrograde labeling, sometimes spanning all layers, were also present in TPO (d,e). In contrast, anterograde labeling in L4 of A1 was largely absent (c), despite strong anterograde and retrograde labeling in supragranular and infragranular layers. These laminar profiles suggested that feedforward and lateral projections from ML targeted $\mathrm{RPB}, \mathrm{CPB}$, and $\mathrm{TPO}$, whereas projections to $\mathrm{A} 1$ were a type of feedback projection.

Further analyses of cortical transport were not pursued in this case, because of uncertainty about the quality of anterograde CTB transport, and some concern that the involvement of the white matter by the injection may have been taken up by fibers of passage to or from the caudal parabelt. However, as the overall projection patterns were highly similar to those observed in subsequent cases, the data have supplementary value. Further, as
CTB is a highly sensitive retrograde tracer, this case served as an important control for the weaker retrograde transport associated with the BDA and FR tracers noted in the other cases.

\section{CASE 2: ML AND CPB INJECTIONS}

The FR and BDA tracers used in this study produced both retrograde labeling of neuronal somata and punctate anterograde labeling of axons and terminals. Typically, labeled terminals were distributed regularly along axon segments, often oriented horizontally (parallel to pial surface and laminae), crossing several cell columns. Some of these axons could be followed for several millimeters, and tended to be more common in L6 and white matter. Diagonal and vertical (radial) orientations were less common, and could occur in any layer. Occasionally, a radially-oriented axon traversed multiple layers, spanning infra and supragranular domains.

Figure 3 depicts sections at different magnification from Case 2 , in which BDA-labeling from injection of CPB was made fluorescent by reacting the sections with streptavidin tagged with a green fluorescent marker (AlexaFluor 488). This allowed simultaneous viewing of terminals from the FR injection of ML (red) and $\mathrm{BDA}$ injection of $\mathrm{CPB}$ in the same section (NeuN fluorescence for laminar identification not illustrated, but see Supplementary Figure 1). In panels A and B, sections from caudal (at level of A1) and rostral (at level of R) locations were selected for illustration. The panels below (C-E) are higher 


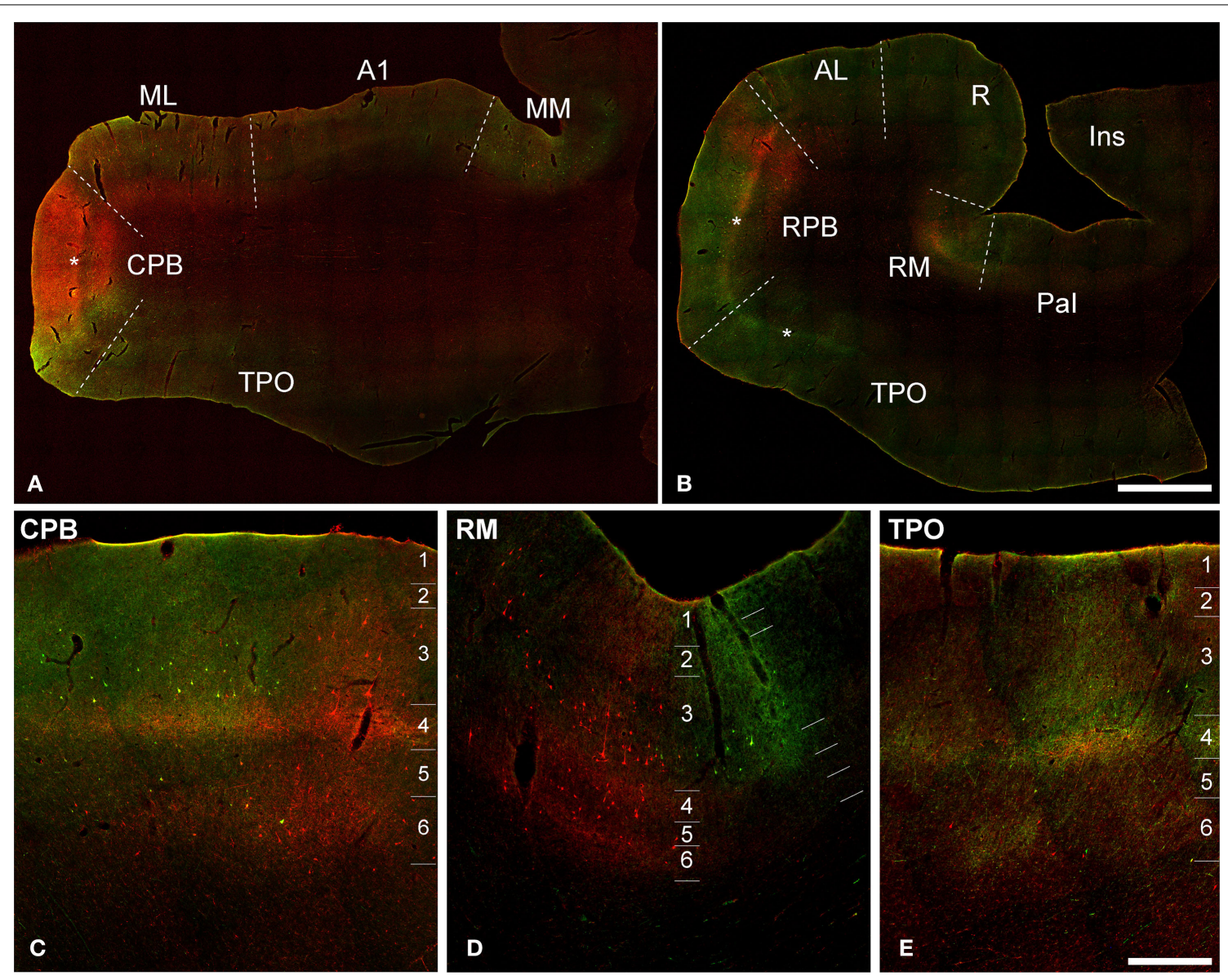

FIGURE 3 | Dual fluorescence images of BDA (green) and FR (red) labeling of axons, terminals, and somata in Case 2. (A,B) Low magnification sections from caudal (A) and rostral (B) sections. Asterisks denote position of layer 4. (C-E) Higher magnification views through portions of CPB, RM, and TPO show overlapping BDA and FR signals. Note overlapping bands in L4 and L6 from both tracers, and patchy columnar labeling across layers in all panels. Scale bars: (A,B) $2 \mathrm{~mm}$; (C-E) $500 \mu \mathrm{m}$. magnification views through portions of $\mathrm{CPB}, \mathrm{RM}$, and TPO where overlapping signals from both tracers are clearly visible. These panels reveal zones of overlapping and non-overlapping transport of FR and BDA to L3 and L4 of these areas. These results illustrate the expected topographic differences between two different areas of auditory cortex, but also reveal a high degree of overlap in L4 and L6, for example, which implies that both input sources (ML and $\mathrm{CPB}$ ) impact activity in the same cortical columns.

Note that, as in Figure 2, anterograde tracer deposits were dense enough in some locations that their laminar distributions were easily discerned even in low magnification images. Otherwise, labeled terminals and axons could only be resolved and visualized at higher magnification. In the figures of cases 2 and 3 that follow (Figures 4-9), anterograde terminal labeling is illustrated at lower and higher magnification for visualization of the details most relevant to this study Graphical representations of the projection patterns for each injection were also prepared to summarize this aspect of the results (Figure 10). Plots and cell counts of retrograde transport in this case are summarized in Supplementary Figure 2 and Supplementary Table 2.

\section{Projections of area $M L$}

The principal outputs of caudal ML reached core, belt, and parabelt areas along the entire rostral-caudal extent of the AC (Figures 4, 5, 10). Terminal labeling differed between areas and between layers. The strongest projections targeted areas at the same rostrocaudal level as the injection and areas just rostral to it.

Rostral to the injection, the most prominent projections from ML reached RPB, AL, RM, RTM and ProA (Figures 4A-D, $\mathbf{5 A}, \mathbf{C}, \mathbf{F})$. These were characterized by lateral and feedforward style inputs, usually with foci in L4 and L6, which stood out from somewhat reduced density in other layers. Most surprising were the strong projections to RM and RTM, as these areas were not expected to be important targets of feedforward projections by ML. These inputs spanned all layers, with prominent foci in L4 and L6 that extended medially into Pro in the floor of the circular sulcus. Weaker projections also reached the rostral core area, R, and rostral A1 where a few branching axons were located in L1/2 and L6. Otherwise, in A1 (Figure 5K), labeled terminals extended in $\mathrm{L} 1 / 2$ across the entire width of field from ML to MM. There was no significant input to RT or rostral TPO, and very sparse inputs to L1 of RTL. 


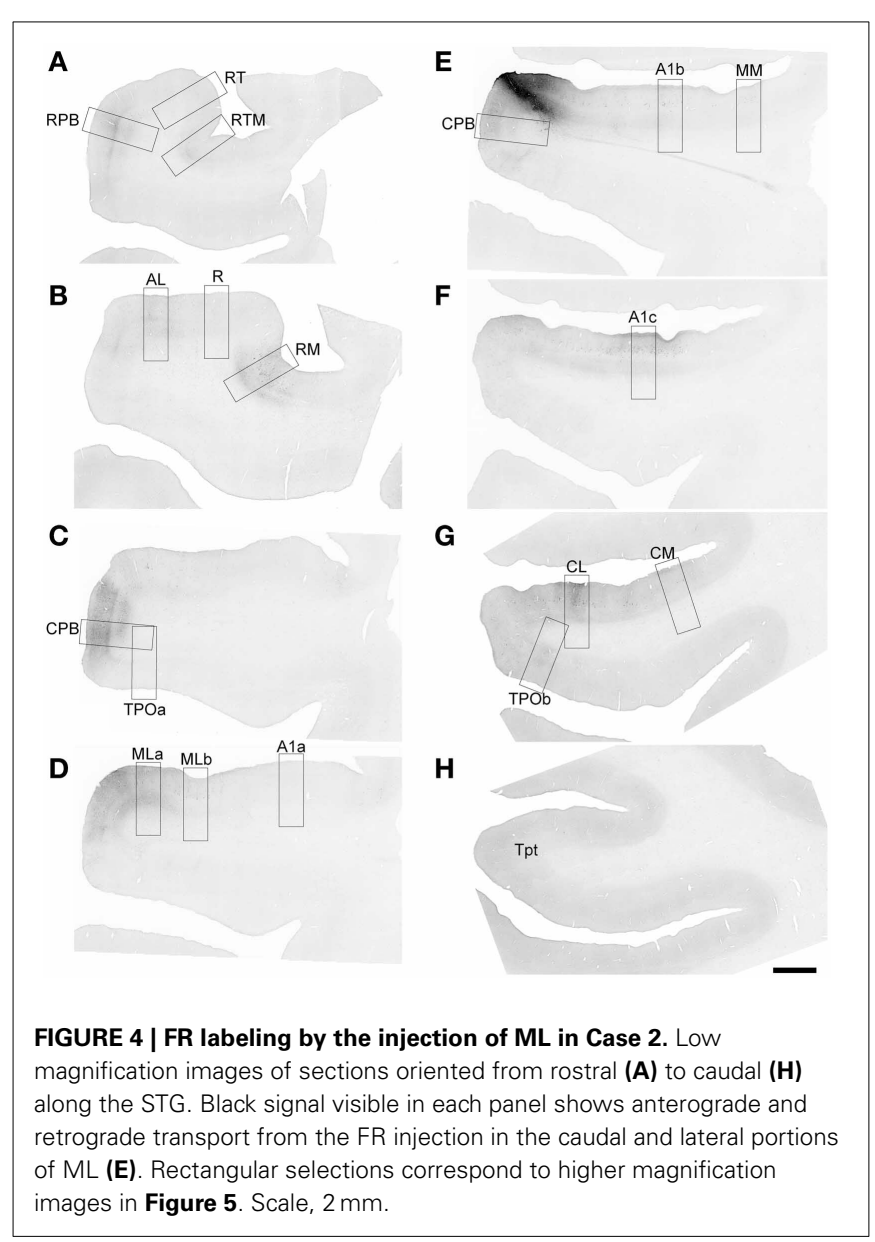

At the approximate rostrocaudal position of the injection (Figure 4E), lateral connections with A1 were most dense in the $\mathrm{L} 1 / 2$ band (Figures $\mathbf{5 M}, \mathbf{O}$ ), moderate in the layers beneath, and sparse in L4. In MM, terminals were mainly found in bands spanning L1/2 and L5/6 (Figure 5N).

Caudal to the injection, terminal labeling was found in CL, $\mathrm{CM}, \mathrm{CPB}$, Tpt, and TPO (Figures 4F-H). The strongest labeling in auditory cortex was in CL, where patches of dense terminal labeling extending across layers were bounded by patches of lesser labeling in L1-3b and L5-L6 (Figure 5Q). Light terminal labeling was found in L4. The laminar distributions in the intervening patches were comparable, but density was reduced overall across layers. In CM, labeling was patchy, as in CL, but not as dense (Figure 5R). In some ways, the laminar patterns in CM resembled those of A1, with heaviest concentrations in L1. Unlike A1, however, was moderate terminal labeling that extended across L2 and L3a, then became very sparse in L3b-6. In CPB caudal to the ML injection, terminal labeling was moderate in supraand infragranular layers, but avoided L3b and L4. In Tpt, very light terminal labeling, continuous with $\mathrm{CL}$ and $\mathrm{CPB}$, persisted onto its gyral and planar domains, but was primarily restricted to axonal branching in L1-3a. Retrogradely labeled cells were mainly located in L3b of CL and CM, with some neurons in L3a and L5/6 of these areas. These results indicate that caudally-directed projections from ML reach the caudal belt areas and Tpt, and are clearly biased toward the supragranular layers (L1-3b). Terminal labeling in L4 was light in CL, sparse in CM, and absent from Tpt, indicating that caudally-directed feedforward projections from ML are extremely limited. The retrograde labeling suggests that ML may be a recipient of feedforward projections from CL and CM, however.

Projections from ML to TPO on the upper bank of the STS were primarily confined to its caudal half (Figure 4G), and roughly in line with the rostral-caudal span of the CPB. These inputs were characterized by two basic laminar patterns, often visible in the same section: (1) full columns of labeled terminals that spanned all layers (Figure 5P), or (2) terminal foci in L1/2, L4, and L6 with very sparse label in the intervening layers. This laminar pattern often occurred in the zones, or patches, that separated those with the columnar labeling pattern. In rostral sections, in line with the RPB, light terminal labeling continued from the ventral RPB for a short distance into the lateral edge of TPO before ceasing entirely. Therefore, ML has direct lateral and feedforward projections to mainly the caudal sector of TPO that bypass the CPB.

Two other patterns of interest concerned the projections to RPB and AL from ML. First, terminal labeling in RPB was concentrated in the dorsal half of the field, with sparse inputs to the ventral half (Figures $4 \mathbf{A}, \mathbf{B}$ ). In contrast, the projections to $\mathrm{CPB}$ from $\mathrm{ML}$ were more uniform from dorsal to ventral across the STG surface, then weakened near the transition to TPO in the STS (Figures 4C-E). The concentration of labeling in the dorsal CPB may reflect topographic gradients, but also may conform to a possible cytoarchitectonic boundary, separating the dorsal and ventral RPB. This possibility has been documented on the basis of architectonic features (Saleem and Logothetis, 2012).Second, in AL, terminals are located in a continuous band involving L1-3a that extended to the lateral edge of the core area, R (Figure 4B). Vertical patches of labeling spanning multiple cell columns were also located in AL (Figure 5D). These were characterized by somewhat higher concentrations of terminals in L4 and L2/3a. Thus, there are inputs to L1 across all of AL with patches characterized by feedforward and lateral inputs.

Altogether, the projections of area ML are consistent with the flow of information along the core-belt-parabelt axis, feeding also into TPO, and also along the caudal-rostral axis within auditory cortex.

\section{Projections of $\mathrm{CPB}$}

With a few notable exceptions, the principal outputs of the ventral CPB injection were very similar to that of caudal ML, reaching nearly all of the core, belt, parabelt areas along the entire rostralcaudal extent of the AC, as well as TPO and Tpt (Figures 6, 7, 10). The span of the $\mathrm{CPB}$ projections covered a larger range along the rostro-caudal axis than those of $\mathrm{ML}$, and therefore a greater number of sections are illustrated at low magnification in Figure 6.

Rostral to the injection site, the main targets of feedforward and lateral projections from the CPB were RPB, RTM, RM, ProA, and TPO (Figures 6A-H). These projections typically 


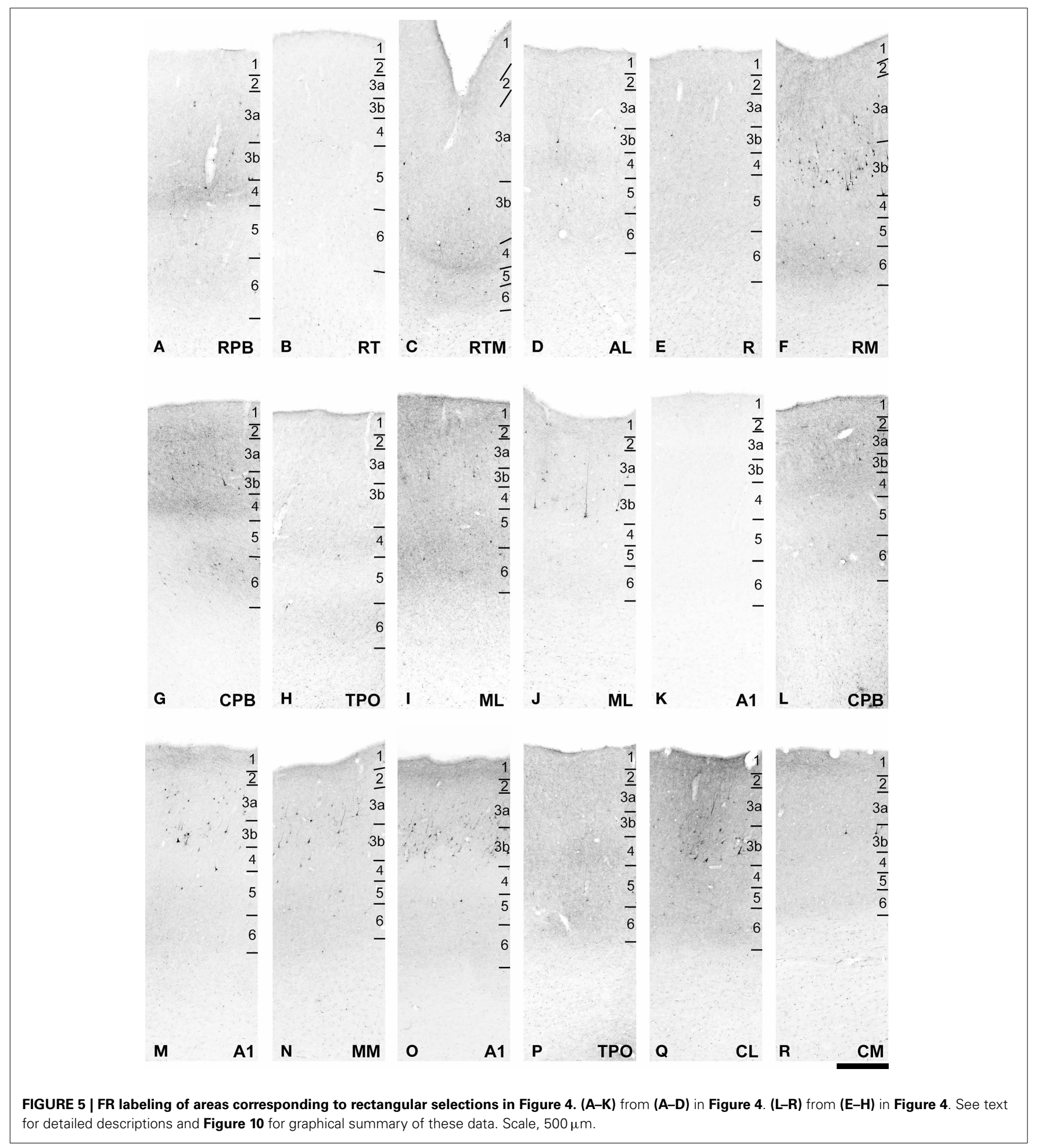

formed prominent bands of terminal labeling in L1/2, L4 and L6, with labeling of variable density in other layers. In some locations, most notably TPO, radial columns of anterograde labeling spanning all layers added to these horizontal bands (Figures 7A,C,D,E,H). These columns were separated by zones with reduced label in L3 and L5. Weaker projections to rostral areas reached $\mathrm{AL}$, and primarily L1-3a and L6, and also the core area, $\mathrm{R}$, where inputs were restricted to a continuous band in L1. As observed for area ML, the weakest CPB projections in the rostral direction were to RTL, where only an occasional axon segment was found in L1. No projections were found to the putative core area, RT. 


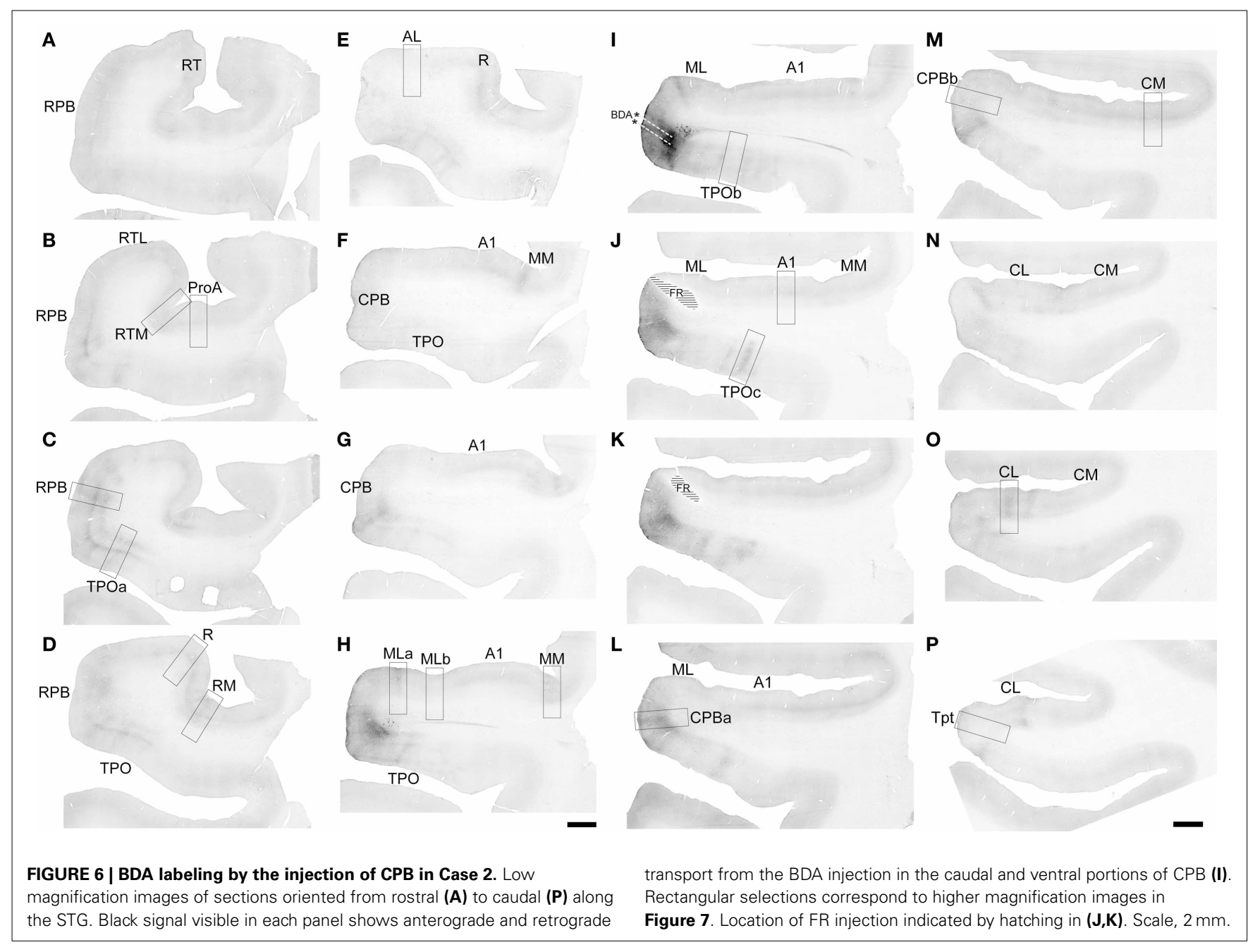

At about the same rostrocaudal level of the injection (Figures 6H-J), strong labeling across all layers formed columns in TPO, CPB, ML, MM, and CL (Figures 7H-K). As in more rostral locations, these dense projections occurred in patches, between which labeling density was significantly reduced. As an example, images of two adjacent patches from area ML are illustrated in (Figures 7H,I). In the left panel $(\mathrm{H})$, terminal labeling spans all layers, and was most dense in L1/2. In the right panel (I), terminal labeling was reduced and mainly found in L1/2 and L6. The visible band in L4 is mainly produced by non-specific background staining, as only a few labeled terminals were found there.

Also at this rostrocaudal level, note that projections to $\mathrm{A} 1$ from CPB were limited to L1, which typically formed a continuous band that ran across the entire lateral to medial extent of A1 at all levels (Figure 7L). Combined with similar projections to L1 of $\mathrm{R}$, it appears that this part of CPB projects evenly to L1 across the entire surface of $A 1$ and $R$ in the core. Projections to MM were concentrated in L1-3a and L5-6, but very sparse to absent in L3b-4.

Caudal to the injection site, terminal labeling was present in the belt areas, Tpt, and TPO. In CPB (Figure $7 \mathrm{~N}$ ), terminal

labeling was mainly located in L1-3a and L5-6, but very sparse in L3b and 4. Further caudal in CPB, the laminar pattern was maintained, but projection density was reduced (Figure 7O). In CL, dense patches of labeled terminals spanning all layers occurred in patches (Figure 7Q), but also zones of lighter labeling concentrated in L1/2 and L6 were present between patches in CL (Figures 6O). In CM (Figure 7P), terminal labeling was primarily in L1/2 and L6, similar to the lighter zones in CL. In caudal TPO, the patchy columnar labeling seen in rostral TPO and other areas continued across at least the lateral $2 / 3$ of the upper bank (Figure 7K) of the STS before diminishing at the caudal level of Tpt (Figures 6I-P). In Tpt, anterograde projections mainly targeted L1-3a (Figure 7R).

Together, these data indicate that except for RT and RTL, CPB has some type of projection to all areas of the auditory cortex, including the core. Feedforward and/or lateral projections mainly target rostral belt and parabelt areas, TPO, and the lateral belt areas adjacent to CPB. Varieties of feedback projections were more typical of the core and caudal medial belt areas. As noted for ML, these projections are consistent with prominent paths of information flow along the core-belt-parabelt-TPO and caudal-rostral axes in the auditory cortex. 


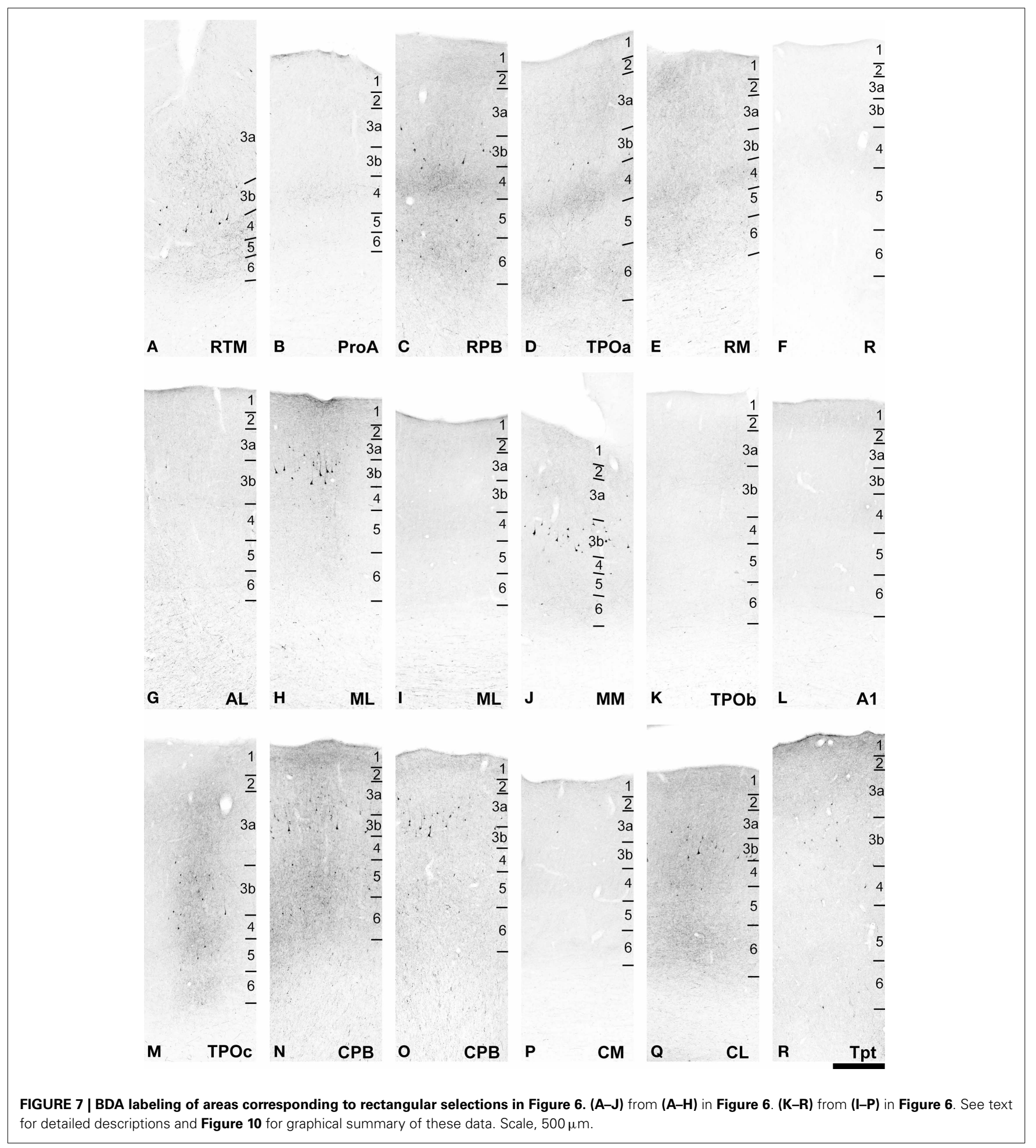

\section{CASE 3: CM INJECTION}

In Case 3, a BDA injection was placed into the rostral and medial portion of CM, near its junction with MM (Figures 8E,F). Overall, anterograde and retrograde transport was very strong to the caudal portion of auditory cortex and uniformly weak or absent to rostral areas.
Rostral to the injection site, BDA labeled axons and terminals were found in $\mathrm{Al}, \mathrm{ML}, \mathrm{MM}$, and $\mathrm{CPB}$, but not in any of the rostral core, belt or parabelt areas. A few labeled somata were noted in the insula of the most rostral section illustrated (Figure 8A, open symbols), at the level of R, but no labeled axons or terminals were found at this level in any field. In rostral A1 


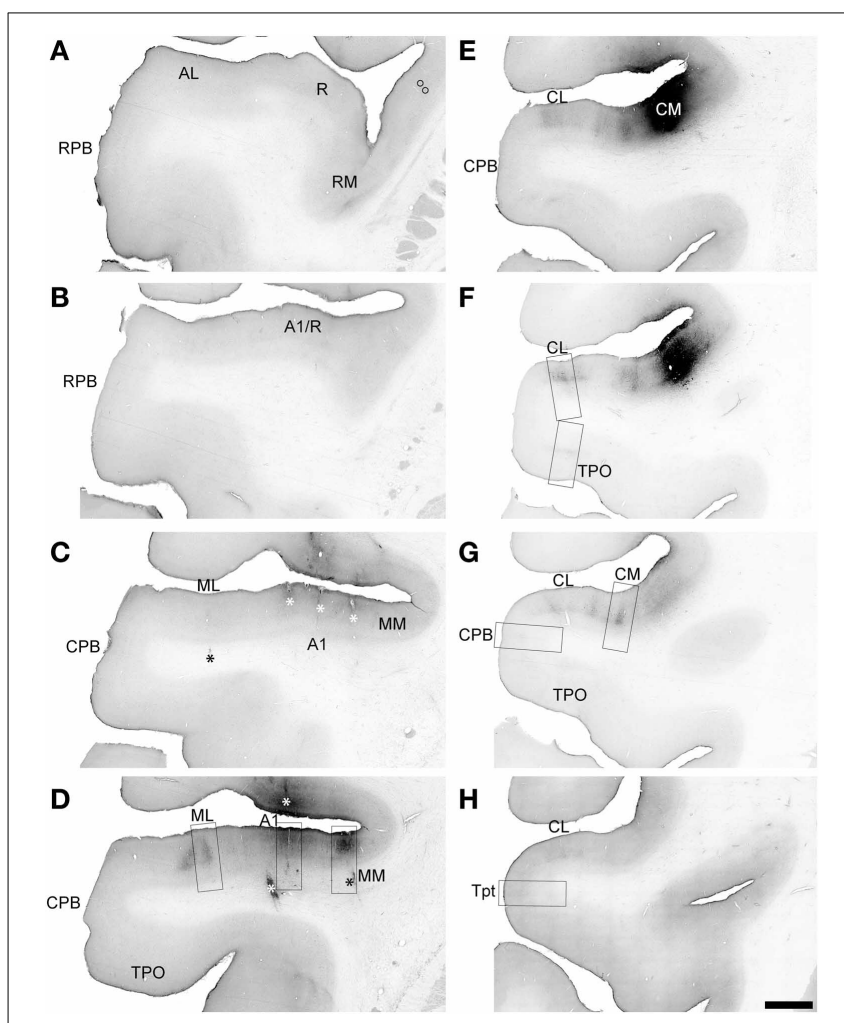

FIGURE 8 | BDA labeling by injection of $\mathbf{C M}$ in Case 3 . Low magnification images of sections oriented from rostral (A) to caudal $(\mathbf{H})$ along the STG. Black signal visible in each panel shows anterograde and retrograde transport from the BDA injection in the rostral and medial portions of $\mathrm{CM}(\mathbf{E}, \mathbf{F})$. Rectangular selections correspond to higher magnification images in Figure 9. Open symbols in (A) indicate location of labeled cell somata in the insula. Asterisks in $\mathbf{( C , D )}$ denote locations of electrolytic lesions. Scale, $2 \mathrm{~mm}$.

and the transition from MM to RM (Figures 8B,C), a small number of axons and terminals were found in L1-3 (not illustrated). Caudally (Figures 8D, 9B,C) labeling in A1 and MM was fairly high in L1, moderate in L2-3b, then light in L4-6. In ML, labeling was found in patchy columns, where terminal labeling was concentrated in L3a-4, and light in L1-2 and L5-6 (Figure 8A). In $\mathrm{CPB}$ rostral to the injection site, only a few isolated axons were found scattered in L3.

Caudal to and in line with the injection site in CM, terminal labeling in CL and other portions of CM formed patchy columns that were distributed throughout the territory covered by both fields (Figures 8F,G, 9D,E). In these columnar patches, labeled terminals were found in all layers, but the distribution was uneven. The greatest concentrations of terminals were in L3b and 4, with lighter labeling in L1-3a and weak labeling in L5-6. The patches were typically linked by reduced axon and terminal density in all layers, but a continuous band in L4 remained prominent, visible even at low magnification (e.g., Figure 8G, area CL). Although not entirely visible in Figure 8G, the L4 band was fairly continuous from CM to CL and into CPB on the STG. The terminal labeling in L4 of CPB (Figure 9G) was much lighter compared to the patches in $\mathrm{CM}$ and $\mathrm{CL}$, but comparable to labeling in

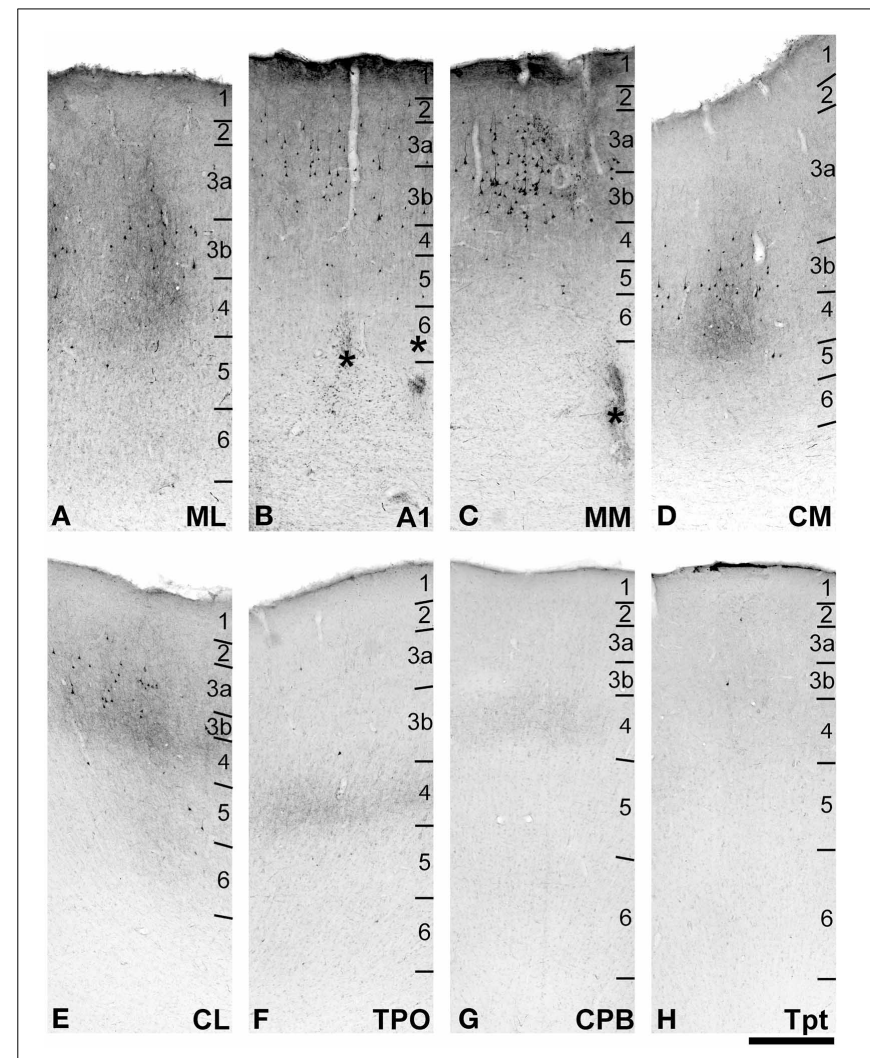

FIGURE 9 | BDA labeling of areas corresponding to rectangular selections (A-H) from (D-H) in Figure 8. Asterisks in (B,C) denote locations of electrolytic lesions. See text for detailed descriptions and Figure $\mathbf{1 0}$ for graphical summary of these data. Scale, $500 \mu \mathrm{m}$.

the intervening zones between patches. In Tpt, a few branching axons with labeled terminals were found in L2-3b and L5-6, with a very light band of terminal labeling in L4 (Figure 9H). This laminar pattern was very similar to that observed in CPB caudal to the injection, but with reduced axon density. In caudal TPO (Figure 9F), terminal labeling was concentrated in L4, with sparse labeling in other layers.

Although the spread of projections from CM was more constricted than for ML and CPB, the areal and laminar projection patterns were consistent with information flow along the same major anatomical axes. We are not certain whether the restricted projections, especially in the rostral direction, exemplify the projections of this part of CM, or whether unknown methodological factors limited transport, or perhaps both.

\section{LAMINAR PROJECTION PATTERNS}

The most common laminar patterns observed were: (1) columns of labeled axons and terminals that spanned all layers, but with distinct or prominent bands in L1, L4, and/or L6. This was typical of projections from areas in presumably lower hierarchical stages to one or more areas at a higher stage where feedforward projections were found (e.g., ML and MM to CPB; ML and $\mathrm{CPB}$ to RPB and RM; MM, ML and CPB to TPO); (2) terminal labeling focused in supragranular and infragranular layers that avoided the middle layers, including L3b and 4, corresponding 


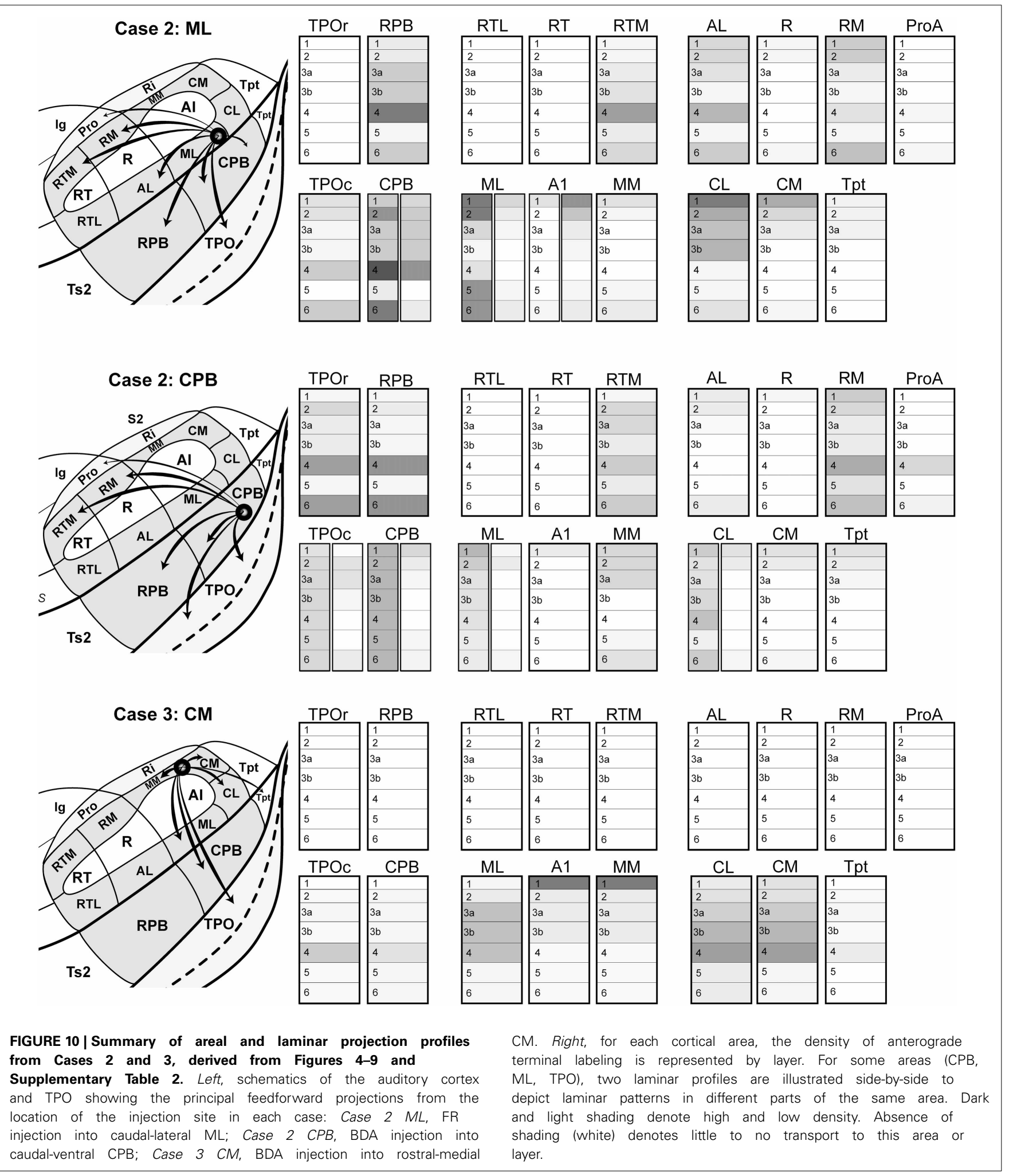

to a lateral type of projection that lacked a feedforward component; and (3) terminal labeling concentrated primarily in L1 or L1-2 (Figure 10, right panels). This was typical of projections to $\mathrm{A} 1$ and $\mathrm{R}$ from $\mathrm{CPB}$ and $\mathrm{ML}$, for example, implying a feedback style of input from higher to lower stage of processing. Other examples of this type were from $\mathrm{CPB}$ to $\mathrm{CM}$ and portions of CL. A somewhat unusual pattern was observed in the projections from $\mathrm{CPB}$ and $\mathrm{ML}$ to Tpt, which were focused in L1-3a. A 
very similar pattern characterized the projection from $\mathrm{CM}$ to $\mathrm{MM}$ and A1, which favored L1-3b. One other unusual pattern was the projection to ProA from ML and $\mathrm{CPB}$, which produced labeled terminals almost entirely confined to L4 and L6. This is the only area that received inputs that did not also at least have inputs to L1 or other supragranular layers.

\section{RECIPROCITY AND NON-RECIPROCITY OF CONNECTIONS}

Although we did not focus on the patterns of retrograde cell labeling in this study, a few observations are worth noting for future consideration. Most of the locations that received inputs from $\mathrm{CPB}, \mathrm{ML}$, or $\mathrm{CM}$ also contained retrogradely labeled cells that project back to the injection site. Generally, these labeled somata were located in L3, and less often in L5 or 6. The absence, or near absence, of retrogradely labeled cells was noted in the connections between several areas. Examples included: (1) CPB to A1, R, ProA, and some portions of CM; (2) ML to A1, R, ProA, and Tpt; and (3) MM to TPO, CPB, and Tpt (Supplementary Figure 2 and Supplementary Table 2). Weaker retrograde labeling was also frequently observed in the territory between columns in which dense terminal labeling across layers was accompanied by numerous retrogradely labeled somata concentrated in L3. The lack of labeled somata in these patches, or zones, was most obvious in the projections to TPO where terminals labeled bands in L1/2, L4, and L6 that joined the more prominent columnar patches. These intervening zones or patches are, therefore, sites which receive inputs from the injected areas, but may not project back to those sources.

The absence of labeled neurons in a location may be significant, but the relative rarity of retrogradely labeled cells in L5 and 6 is of questionable validity, as this would imply that most of the reciprocity between connected areas is accomplished via the connections of L3 neurons. This is unlikely. One possible explanation is that this reflects a technical artifact. It is widely known that $10 \mathrm{kDa} \mathrm{BDA}$ does not produce extensive retrograde labeling of neurons. Further, we have noted in prior studies of macaque auditory cortex that retrograde transport of the fluoruby and fluoremerald dextran tracers tends to be biased toward supragranular neurons for some unknown reason (Smiley et al., 2007). On one hand, in all of the areas where significant concentrations of terminals were found, retrogradely labeled cells were also present. By that definition, we could surmise that those interareal connections were reciprocal. However, the labeled cells were usually concentrated in L3, with fewer cells in L5 or 6, which may be an underrepresentation of the actual projection. We would add here that in contrast to cases $2-3$, the CTB injection of ML in Case 1 (Figure 2) produced robust retrograde labeling of supragranular and infragranular neurons in most areas, which provides additional support for the biased transport conjecture. Therefore, although the absence of labeled somata in an area or layer may accurately reflect the absence of a connection, we cannot be entirely certain. For this reason, we elected not to emphasize the retrograde connection patterns in this study. For reference, however, plots and cell counts for Case 2 are summarized in Supplementary Figure 2 and Supplementary Table 2.

\section{DISCUSSION}

The purpose of the present study was to explore the laminar projections of selected caudal belt and parabelt areas, pursuant to a complete survey of the laminar projections of each area of the macaque monkey auditory cortex. Because prior studies and our current models of auditory cortical organization are based primarily on the analysis of neuronal somata labeled by retrograde transport, surprisingly little is known about the laminar circuitry of auditory areas within the superior temporal region. From those foundational earlier studies, low resolution wiring diagrams were generated, which now form the basis of our working models of auditory cortex organization in primates (Hackett et al., 1998; Kaas and Hackett, 1998, 2000; de la Mothe et al., 2006; Hackett, 2011). These diagrams depict connections between areas using lines and arrows (Figure 1), but lack information about the laminar projections of these areas. Therefore, much remains to be learned about these circuits by generating high-resolution wiring diagrams of the laminar circuitry, noting that such models were generated long ago for the visual cortex (Felleman and Van Essen, 1991), and continue to be refined (Markov and Kennedy, 2013). Looking ahead, development of these models is essential for generating and testing meaningful and informed hypotheses about auditory cortical function. Although limited in scope, the present study yielded several new discoveries of sufficient importance to compel modifications of our working model, as discussed below. These small steps increase our motivation to greatly expand this line of inquiry, as additional modifications of the model may result.

To summarize the present findings (Figure 10), the laminar projection patterns of the caudal belt and parabelt support and extend the hypothesis that information flows along two major axes in auditory cortex: core-belt-parabelt and caudal-rostral. First, projections with feedforward characteristics are directed from the caudal belt areas (ML, CM) to caudal domains of the parabelt $(\mathrm{CPB})$ and TPO. The CPB also projects in this manner to caudal TPO. These patterns are consistent with a stream of information flow directed along the core-belt-parabelt axis of auditory cortex, that also feeds strongly into TPO from two different stages of the hierarchy (caudal belt and parabelt). Second, feedforward patterns were also evident in the projections of the caudal belt and parabelt to rostral belt and parabelt areas and rostral TPO. Some of these areas even received overlapping inputs from caudal belt and parabelt (e.g., RPB, RM, and RTM). Overall, these patterns are consistent with a flow of information from caudal to rostral among auditory and related areas in the superior temporal region (Hackett, 2011). Overlaid on these two major patterns of projections were the more complex area-specific projection patterns, for which the laminar relationships were more variable. The balance of the discussion highlights some of the more interesting details, which are presented in the context of a revised model of auditory cortical wiring.

\section{SIMILARITIES AND DIFFERENCES IN THE PROJECTIONS OF ML, CM, AND CPB}

The similarities and differences in the connections of ML, CM, and $\mathrm{CPB}$ were enlightening with respect to general patterns of information flow and differences between individual areas. Perhaps the most robust finding was that ML and CPB have comparable laminar patterns of feedforward and lateral projections to several of the same belt and parabelt areas located rostral to or in line with the location of their injections (e.g., rostral: RPB, RTM, 
RM, ProA; caudal: CPB, and caudal TPO) (Figure 10). Rostral $\mathrm{CM}$ targeted some of the same areas (CPB and caudal TPO). These projections often spanned all layers, but were usually characterized by prominent bands of terminal labeling in L1/2, L4, and L6. Overall, these results indicate that the outputs of ML, $\mathrm{CM}$, and $\mathrm{CPB}$ are directed along the two major anatomical axes within auditory cortex (core-belt-parabelt and caudal-rostral). The forward-directed projections along these axes have a prominent serial component that extends into TPO, but the projections to this region are not strictly serial since outputs from both belt and parabelt areas directly reach this field. These connections are discussed further below.

Other similarities were the laminar patterns of the projections that ran against the dominant feedforward gradients. Projections from ML and CPB to areas caudal to their injection sites (MM, $\mathrm{CM}, \mathrm{CL}$, caudal CPB, Tpt) were biased toward supra- or supraand infragranular layers, avoiding the middle layers, reflecting feedback or lateral connectivity. Similarly, projections to the core areas, mainly A1 and R, generally avoided the middle layers and were often concentrated in L1. Similarly, the projections of CPB to most of the belt areas also avoided the middle layers. These patterns indicate that feedback types of projections tend to characterize information moving in the opposite direction along the major axes. Elements of these patterns have been variably noted in prior studies (Galaburda and Pandya, 1983; de la Mothe et al., 2006).

In terms of differences between the injected areas, a few are highlighted here. First, ML had feedforward and lateral projections to AL, but the projections from $\mathrm{CPB}$ to AL reached supraand infragranular layers only. This indicates that $\mathrm{AL}$ is in the line of rostrally-directed feedforward projections from ML in the caudal belt, but not from the caudal parabelt. The absence of feedforward inputs to AL from $\mathrm{CPB}$ is consistent with straightforward core-belt-parabelt hierarchical relationships (Hackett et al., 1998). A second notable difference was that CPB had dense feedforward and lateral projections to caudal and rostral TPO, but projections to rostral TPO from ML were sparse to absent. This is intriguing since ML had strong forward projections to auditory areas at that rostral level (i.e., RPB, RM, and RTM), but not rostral TPO. It may turn out that $\mathrm{CPB}$ is the only caudal area with significant projections to rostral TPO. We predict that some or perhaps all of the rostral belt and parabelt areas will target that area, however. A third difference was that, whereas forward directed projection from the rostral $\mathrm{CM}$ injection targeted some of the same areas as ML and CPB (i.e., CPB, caudal TPO), projections were also concentrated in the middle layers of $\mathrm{ML}, \mathrm{CL}$, caudal portions of CM, and weakly in gyral Tpt. This suggests that some feedforward projections are directed laterally and caudally from the rostral CM position. Evidence of caudally-directed information flow has been observed in some studies (de la Mothe et al., 2006), but the data remain thin and will require further study of the caudal and medial belt areas. Finally, the absence of projections to all rostral auditory areas from this CM injection was striking. We are not certain whether technical factors could account for this, as projections from this region to rostral locations were noted in marmosets and macaques (de la Mothe et al., 2006; Smiley et al., 2007).
Finally, the concentration of inputs to L1/2, L4, and L6 indicate that, in addition to classic feedforward projections (to L4), significant inputs also terminate in other layers. This implies that within the bundle of projections from one area to another are multiple "strands" that target neurons in different layers. Although highly intriguing, it is not known whether the signals carried along each of these strands bear the same information or even have the same timing. At present, we do not know the specific cell types or laminar positions of the projecting (source) neurons, only that the majority are pyramidal neurons in layers 3 and 5 . Future anatomical studies should incorporate methods to dissect these details. Ideally, these would be coupled with physiological recordings using laminar arrays to characterize the properties of the signals carried by each of these strands and their impact on neurons in all layers.

In summary, there were prominent similarities in the feedforward, lateral, and feedback projections of the caudal belt and parabelt areas that were injected. These lend support to our hypotheses about information flow in auditory cortex (Hackett, 2011). The results also reveal significant differences in the laminar projections of individual areas. This highlights the notion that each area, and likely each layer, has a different functional role, and sets the stage for studies that can bring out those features.

\section{DIVERGENT AND CONVERGENT PROJECTIONS BLUR HIERARCHICAL RELATIONSHIPS}

The projection patterns of the caudal belt and parabelt had divergent and convergent characteristics. These patterns were in line with some, but not all, of the hierarchical relationships established in prior studies.

Divergent projections were reflected in two main ways. First, multiple areas of auditory cortex (and TPO) were labeled by injections of each area (Figure 10). The fact that a single area of auditory cortex has connections with several others is not novel, but one worth exploring further, since the present findings indicate that each area can foster feedforward, lateral or feedback projections to several other areas and at more than one level of auditory processing. ML, CM, and CPB had projections to multiple core, belt, and parabelt areas, as well as TPO. Also notable is that none of these tracer injections filled the entire target area. This is interesting since each of the injected areas is large, and its connections could be topographically distributed, meaning that the projections from different loci within a source area may be different in some ways (e.g., tonotopy, binaural integration, inputs from other areas, etc.). It will be important in future studies to compare injections placed in different portions of the same area to reveal whether its outputs are topographically organized. Second, within a single recipient area (e.g., RPB, TPO, A1), the projections from one of the injected areas were typically not contained within a single topographic locus (e.g., point-to-point). Instead, inputs were often distributed over multiple loci with different laminar profiles (e.g., Figure 10, twin panels in CPB, ML, A1, TPOc, CPB, CL). For example, patches that contained columnar labeling across all layers were often separated by inter-patch regions where terminal labeling was concentrated only in L4 and L6. This was common in belt, parabelt, and TPO. In other areas, the inputs were evenly distributed in some layers across much or 
possibly all of the entire field (e.g., continuous labeling of bands in $\mathrm{L} 1$ of $\mathrm{A} 1$ and $\mathrm{R}$ ). These varied patterns of divergence imply that the outputs of a given area are processed in parallel by several areas, and by multiple locations within each of the recipient areas. There is little evidence of point-to-point connectivity in these circuits.

Convergent projections from two or more sites onto a single area or locus within an area were frequently observed. Three key observations are worth noting here. First, the projections of at least two, and sometimes all three, injected areas reached many of the same areas (Figure 10). The main exception, noted above, was rostral TPO, which was only reached by the CPB injection. Second, these projections sometimes originated from different levels of the core-belt-parabelt hierarchy. RPB, RM, RTM, and caudal TPO are examples of recipient areas in which convergent projections originated in belt and parabelt areas. Third, projections to a single area often overlapped in a single column, layer, or layers. An obvious example is in Figure 3, where fluorescent labeling was used to reveal the projections from ML and CPB in the same sections from Case 2. Overlap was substantial in L4 and L6 of CPB and TPO. Although CM was injected in a different case and not reflected in Figure 3, the locations of its projections to L4 of the CPB and TPO (Figures 8, 9) rather strongly suggest that its inputs would also be overlapping. Altogether, these patterns imply that each area, specific layers within each area, and even multiple columns within each area process convergent inputs in parallel from two or more other auditory cortical areas at different hierarchical levels.

The functional implications of such widespread divergence and convergence are not very clear, as there are minimal data on the differences in neuronal response properties between hierarchical areas. Recent neurophysiological data from our laboratory provide some room for speculation. In recordings from 10 core, belt, and parabelt fields, an increasing gradient in response (spike) latencies was observed along the core-belt-parabelt and caudalto-rostral axes (Camalier et al., 2012) in response to clicks, tones, and noise bursts The gradient was strongest from caudal to rostral areas, and weakest from belt to parabelt. The results of this study, and several others with related findings, generally support the notion that feedforward signal flow is directed along the two major anatomical axes. However, we also noted that mean response latencies were only slightly longer in parabelt vs. lateral belt areas, and that their distributions were highly overlapping. When considered alongside the present study, one could predict that the information delivered to an area, or specific location within an area, by convergent projections from ML and CPB may arrive within a narrow temporal window. This would apply to several areas, based on the present study (RPB, RM, RTM, caudal TPO). Although the nature of the information delivered to a given site through convergent inputs is presumably distinct, those signals could reach that site at about the same time. This is especially intriguing since multiple areas at different hierarchical levels appear to receive at least some convergent inputs from different levels. In addition, for a given site, the precise timing of these events could vary between input layers. As one example, earlier arriving (e.g., modulatory) inputs to L1 could set the tone for later arriving (e.g., driving) inputs to L4, or perhaps reset the phase of ongoing oscillations (Lakatos et al., 2005b).

Along these lines, then, an important future line of inquiry will be to explore the possibility that each of these diverging and converging strands has different functional properties. It is likely that converging inputs from several areas are distinct, but are the divergent projections from one site propagating the same signal in parallel to multiple others? Do those signals differ by the laminar position and cell type of both source and target? These details are essential for understanding the ways in which signals are processed and distributed between areas of auditory cortex.

\section{FEEDBACK PROJECTIONS TO THE CORE FROM THE PARABELT}

It has been frequently observed in previous studies in primates that the parabelt does not receive significant input from the core areas A1 or R, and sparse inputs from RT. Projections to the parabelt from within the auditory cortex arise almost exclusively from the belt areas. This is the key anatomical support for a core-beltparabelt hierarchy (Hackett et al., 1998). However, as previously noted in macaques (Pandya and Rosene, 1993) and marmosets (de la Mothe et al., 2006), the parabelt region does appear to have a significant projection back to L1 of the core. In the present study, our results affirm those observations, and also indicate that those inputs are broadly distributed over the core (Figure 10, laminar profiles). The ventral CPB location injected in Case 2 projected evenly to $\mathrm{L} 1$ across the entire surface of $\mathrm{A} 1$ and $\mathrm{R}$, although input to L1 of the putative third member of the core region, RT, was very sparse. The broad spread of the L1 projection over the core is very similar to that noted by Pandya and Rosene (1993) after a large isotope injection of the STG that probably involved CPB and ML. If we assume this pattern to be true of the parabelt region in its entirety (rostral and caudal divisions), then the feedback projections to the core would form an expansive and dense matrix over the entire core region that lacks obvious rostrocaudal topography. Given that apical dendrites from subpopulations of cells in almost all layers ramify in L1, the projections to L1 from even a single location in the parabelt could exert a powerful influence over global activity within the core region.

\section{IS THE ROSTRAL MEDIAL BELT A CONNECTIONAL CROSSROADS?}

In our studies of the connectivity of the auditory cortex in primates, we have noted that the rostromedial belt area, RM, is broadly connected with rostral and caudal auditory areas in a manner distinct from other belt areas (Hackett et al., 1998; de la Mothe et al., 2006; Smiley et al., 2007). See also Galaburda and Pandya (1983) (Figure 10). Retrograde tracing studies showed that whereas the caudal and rostral belt areas tend to have stronger connections with other caudal and rostral areas, the connections of RM appeared to lack such topography. Thus, the outputs of $\mathrm{RM}$ are more broadly distributed to belt and parabelt areas along the caudal-rostral axis. The surprising results of the present study add to this quandary, by revealing that the caudal belt and parabelt are sources of strong convergent inputs to RM that have both feedforward and lateral features, such as dense terminal labeling in L4, L6, and other layers. We also noted that RTM received similar inputs from the same areas. In contrast, the caudal medial 
areas in this study (MM, CM) did not have these convergent input profiles, implying that there is not a generalized pattern of feedforward or lateral inputs to the medial belt from the lateral belt or parabelt.

\section{INPUTS TO AREA TPT}

The caudal borders of the belt and parabelt regions are bordered by the temporal parietotemporal area $(\mathrm{Tpt})$, which is mostly known for its multisensory features, including auditory responsiveness in some domains (Leinonen et al., 1980). Systematic studies of the neurophysiological properties have not been achieved so far. In prior anatomical studies from our research groups, retrograde tracer injections of Tpt and adjacent belt areas (CM, CL) revealed that its strongest cortical connections included the caudal belt and parabelt regions, whereas connections with the auditory core region are sparse (Hackett et al., 1998; Smiley et al., 2007). The principal thalamic inputs to Tpt include the medial/magnocellular division of the medial geniculate body $(\mathrm{MGm})$ and multisensory nuclei of the posterior thalamus (i.e., suprageniculate, Sg: limitans, Lim; posterior, Po; medial pulvinar, PM), whereas inputs from the dorsal divisions of the MG (MGd) are sparse and variable (Hackett et al., 2007). On the basis of these connections, we have long considered Tpt to be an auditory-related field that is strongly influenced by the caudal belt and parabelt, and other sensory areas.

In the present study, projections to Tpt from $\mathrm{CPB}$ and ML targeted L1-3a, and sparse projections from rostral CM reached L1-4 (Figure 10). In the absence of other data, these patterns raise questions about which, if any, of the auditory cortical areas are a significant source of feedforward inputs to Tpt? The most likely sources would be CL and CM. Although our rostral CM injection revealed only sparse projections to L1-4 of gyral Tpt, perhaps stronger inputs may arise from caudal CM and parts of CL. In the absence of significant feedforward projections to L4 of Tpt, however, it is still possible that the inputs to L1-3a from caudal belt or parabelt areas could significantly impact auditory activity in this area. Given its position in the temporal-parietaloccipital junction, and projections to posterior parietal and dorsal prefrontal cortex (Hackett et al., 1999; Romanski et al., 1999a,b; Lewis and Van Essen, 2000), Tpt is potentially important link between higher order sensory cortex and the targets of the dorsal stream. Detailed studies of Tpt are long overdue.

\section{FEEDFORWARD PROJECTIONS TO TPO FROM CAUDAL BELT AND PARABELT AREAS}

In numerous prior studies, it has been observed that areas of the STG corresponding to the auditory belt and parabelt are broadly connected with areas on the upper bank of the STS corresponding to the TPO. We did not make efforts to subdivide TPO architectonically, but it appears that most of the terminal and cellular labeling was located in the rostral (TPOr), intermediate (TPOi) and caudal (TPOc) divisions ( TPO2-4), which approximately corresponds to the superior temporal polysensory area (STP) in other nomenclature (Jones and Powell, 1970; Seltzer and Pandya, 1978, 1989b, 1994; Cipolloni and Pandya, 1989; Barnes and Pandya, 1992; Cusick et al., 1995; Seltzer et al., 1996; Hackett et al., 1998; Padberg et al., 2003). These studies, which primarily used retrograde tracers, revealed that populations of labeled cells in TPO are rather dense, tend to be clustered in patches, and exhibit some degree of rostral-caudal topography, although there is also substantial overlap of rostral and caudal areas of the belt and parabelt along this axis. Data on the laminar input patterns from anterograde tracers are unfortunately rather scarce. Seltzer et al. (1996) made a large injection of the caudal parabelt, confined to the surface of the STG, that produced patches of terminal labeling along the caudal-rostral extent of TPO in the upper bank of the STS. In a second case, the CPB injection also extended into the upper bank of the STS. In this case, additional terminal labeling was found to extend beyond TPO and the upper bank to the fundus and lower bank, producing label in areas such as MT, MST, and FST. Cusick et al. (1995) also found that injection of the caudal parabelt produced patches of terminal labeling in TPO. In both studies, it was noted that these terminations spanned across layers in columns, were focused on L4, or were mixed. Thus, the laminar patterns they observed are highly similar to those identified in the present study (Figure 10).

Almost all of the existing data reveal that connections with auditory cortex do not extend significantly beyond the fundus of the STS to its ventral bank or to the inferior temporal gyrus (ITG), nor are there any clear connections with the middle temporal (MT) complex, or V5, which is a visual region known to be involved in visual motion processing. Otherwise, TPO and adjacent fields are broadly connected with primary and secondary sensory areas of visual and somatosensory cortex, and higher order areas of prefrontal and posterior parietal cortex (Seltzer and Pandya, 1978, 1989a,b; Pandya and Seltzer, 1982; Ungerleider and Desimone, 1986; Boussaoud et al., 1990; Cusick et al., 1995; Lewis and Van Essen, 2000; Saleem et al., 2000; Padberg et al., 2003; Markov et al., 2014). The connections of parietal and dorsolateral prefrontal cortex tend to overlap in rostral and caudal sectors of TPO, while connections of the posterior parietal and STG tend to be adjacent and non-overlapping (Barnes and Pandya, 1992; Seltzer et al., 1996). An interesting feature of the convergence of inputs in TPO is that they can be patchy, overlapping or interdigitating. It is not yet clear how patches associated with auditory cortex relate to those associated with other cortical fields. Although unimodal, bimodal, and trimodal responses to auditory, somatosensory, and visual stimuli have been recorded in TPO (Benevento et al., 1977; Desimone and Gross, 1979; Bruce et al., 1981; Baylis et al., 1987; Hikosaka et al., 1988; Schroeder and Foxe, 2002), the anatomical data suggest that while sensory inputs to STS may be initially segregated by modality, local connectivity provides a basis for multisensory interactions.

\section{CONNECTIONS BEYOND THE SUPERIOR TEMPORAL REGION}

We did not evaluate the projections to subcortical and other cortical regions for the present study, but in cursory inspections, we did note that the areas injected produced labeling in frontal, medial temporal, and thalamic locations. We did not observe labeling in posterior parietal areas, however, as might have been expected from prior studies (Pandya and Kuypers, 1969; Pandya et al., 1969; Lewis and Van Essen, 2000; Smiley et al., 2007). Because transport was judged to be very good from at least two of 
these injections, we are inclined to conclude that ML and CPB do not have significant projections to posterior parietal areas. Rather, judging from the earlier studies and more recent data (Markov et al., 2014), it is likely that Tpt, and perhaps CM or CL may be the most dominant sources of inputs to posterior parietal areas from superior temporal cortex. Interestingly, one recent study found significant projections between posterior parietal and RPB areas (Markov et al., 2014), suggesting that a more determined survey of these connections is warranted.

\section{INTEGRATION WITH PRIOR STUDIES}

One of the earliest studies of the laminar patterns of projections in auditory cortex was conducted in owl monkeys. Fitzpatrick and Imig (1980) analyzed projections in the core and belt after placing isotope injections into A1 or R. They found that projections from these core areas to belt areas often spanned layers, but sometimes with concentrations in $\mathrm{L} 4$ alone, L3a/4, L3a/4/6. These laminar patterns were similar to those observed in the feedforward and lateral projections between areas in the present study. They also found that within the core, projections from Al to $\mathrm{R}$ targeted L4, consistent with a rostrally-directed flow of information within the core.

Three studies used tracers with anterograde or mixed anterograde and retrograde tracing properties to study auditory cortical connections in marmosets (Aitkin et al., 1988; de la Mothe et al., 2006; de la Mothe et al., 2012). Both sets of studies found that projections from core to belt areas often resulted in columnar terminations spanning layers, but often with a focal band of higher density in L4. In de la Mothe et al. (2006), injections of RM and CM or MM labeled columns of terminals in the lateral belt and parabelt areas, variably punctuated by more intense bands in L2/3a, 4 and 6 . These columns were often separated by columns of weaker labeling, but it was common for dense terminal labeling to persist continuously in the L4 and 6 bands. This suggested that the medial belt areas give rise to feedforward and lateral projections to the lateral belt and parabelt. One additional note, when anterograde terminals were concentrated in L4 of another belt or parabelt area from these injections, they tended to be located in L4 in sites rostral to the injection site, but not caudal. So, in addition to a core-belt-parabelt pattern, there were hints of a rostrallydirected bias in the feedforward projections from the medial belt.

In their foundational study, Galaburda and Pandya (1983) used isotope tracers with anterograde transport properties to study the connections of auditory areas in the macaque monkey STG. Surprisingly, after 30 years, this study stands alone as the most extensive survey of anterograde projections in the auditory cortex of macaques. A major conclusion of that study supported a rostrally-directed pattern of connectivity between areas corresponding to the core, belt, and parabelt regions (terminology transposed to match our nomenclature). These patterns were tied to progressive stages of architectonic differentiation along this axis (caudal to rostral). Although injection sites were typically large, covering more than one field, some general patterns were identified that were also observed and refined by the present study. For example, in case IX, a large isotope injection involving the caudal parabelt and Tpt resulted in feedforward projections to the caudal parabelt and lateral belt, projections to L1 of the core (A1), and terminations across layers in areas corresponding to the RM and MM fields. In cases IV and V, injections of primarily the RPB generated feedforward projections to belt and parabelt areas rostral to the injection site, but predominately L1 projection to caudal CPB. In general, the patterns showed that rostrallydirected projections mainly originate in L3 and terminate in L4 or across all layers in the rostral targets. Caudally-directed projections tended to originate in infragranular layers and terminate in superficial layers. Projections from the core to belt areas were focused on L4, and projections from belt and parabelt to core were focused in L1. Projections from lateral belt to medial belt were spread broadly across layers. Overall, these patterns were generally comparable to those of the present study, although the detailed laminar patterns of connectivity revealed herein varied in a more specific manner between aerial targets, and the greater sensitivity of the tracers revealed the presence of axons and terminals in additional layers.

\section{CAVEATS AND FUTURE DIRECTIONS}

The present study is based on only 4 tracer injections in 3 areas of 3 different experimental cases. Ideally, we would aim to have at least two injections from each target area as a means to evaluate reliability. There is always some variability in transport between injections into cortex. Reliable control of the precise size and location of the injections is generally not possible, even if all experimental variables are exactly repeated. In part, this is because the transport properties of different tracers vary, and the uptake and transport of the same tracer can vary due to factors that appear to be beyond experimental control. For the cases illustrated here, the injections were judged to be very good in terms of placement within a single area, involvement of all layers, and transport, and so we have a high degree of confidence in the results. Clearly, additional studies of this type are desperately needed to obtain detailed and comprehensive wiring diagrams of the projections of all auditory cortical areas, and bolster the findings of the present study. This will be a challenging pursuit, as the territory is relatively large, and many of the areas are buried in locations that require passing through or retracting the cortex of intervening regions. This is a necessary endeavor, however, as our understanding of auditory cortical function depends critically on knowledge of its circuitry. In the meantime, however, the results of this study raised several important questions about the diversity of ways in which signals are passed between areas, layers, and even specific cell types. Many of these questions can be addressed now by using laminar array recording techniques to document the properties of the signals carried by the various strands of projections that reach a given site.

\section{ACKNOWLEDGMENTS}

The authors gratefully acknowledge the support of NIH grants R01DC04318 to Troy A. Hackett, R01DC011490 to Charles E. Schroeder, and R21DC012918 to Yoshinao Kajikawa.

\section{SUPPLEMENTARY MATERIAL}

The Supplementary Material for this article can be found online at: http://www.frontiersin.org/journal/10.3389/fnins.2014. 00072/abstract 
Supplementary Figure 1 | Examples of architectonic features used to delineate areas and layers of auditory cortex. (A) Coronal section at the level of mid-A1 reacted for NeuN IHC (blue fluorescence). Borders between other areas indicated by lines following radial orientation of cell columns. (B) Coronal section in same series as the section in (A), but stained for VGluT2. (C-F) Coronal section through area ML, showing triple fluorescent labeling of FR, BDA, and NeuN. (F) is a grayscale conversion of (E), showing position of layers. (G-J) Coronal section through MM. Same conventions as (C-F). (K-N) Coronal section through area TPO, showing BDA transport and NeuN. Scale bars: (A-N), $500 \mu \mathrm{m}$.

Supplementary Figure 2 | Plots of retrogradely labeled cells in Case 2 following tracer injections into ML (FR, open triangles) and CPB (BDA, filled circles).

\section{Supplementary Table 1 | Gray Level Index (GLI) values of anterograde labeling in auditory cortical areas from tracer injections in Cases $\mathbf{2}$ and $\mathbf{3}$. Measurements were taken from the sections illustrated in Figures 4-9. For each injection, GLI values are sorted by area and layer. Letters A-R in the top row correspond to panel numbers in Figures 5, 7, 9. These data are summarized graphically in Figure $\mathbf{1 0 .}$}

Supplementary Table 2 | Retrograde-labeled cell counts and percent of total cells from tracer injections into ML (FR) and CPB (BDA) (Case 2).

Sorted by cortical area in supragranular (S) and infragranular (I) layers. The results are graphically summarized in the charts below and in

\section{Supplementary Figure 2.}

\section{REFERENCES}

Aitkin, L. M., Kudo, M., and Irvine, D. R. (1988). Connections of the primary auditory cortex in the common marmoset, Callithrix jacchus jacchus. J. Comp. Neurol. 269, 235-248. doi: 10.1002/cne.902690208

Bannister, A. P. (2005). Inter- and intra-laminar connections of pyramidal cells in the neocortex. Neurosci. Res. 53, 95-103. doi: 10.1016/j.neures.2005. 06.019

Barbas, H. (2007). Specialized elements of orbitofrontal cortex in primates. Ann. N.Y. Acad. Sci. 1121, 10-32. doi: 10.1196/annals.1401.015

Barnes, C. L., and Pandya, D. N. (1992). Efferent cortical connections of multimodal cortex of the superior temporal sulcus in the rhesus monkey. J. Comp. Neurol. 318, 222-244. doi: 10.1002/cne.903180207

Baylis, G. C., Rolls, E. T., and Leonard, C. M. (1987). Functional subdivisions of the temporal lobe neocortex. J. Neurosci. 7, 330-342.

Bendor, D., and Wang, X. (2008). Neural response properties of primary, rostral, and rostrotemporal core fields in the auditory cortex of marmoset monkeys. J. Neurophysiol. 100, 888-906. doi: 10.1152/jn.00884.2007

Benevento, L. A., Fallon, J., Davis, B. J., and Rezak, M. (1977). Auditory-visual interaction in single cells in the cortex of the superior temporal sulcus and the orbital frontal cortex of the macaque monkey. Exp. Neurol. 57, 849-872. doi: 10.1016/0014-4886(77)90112-1

Boussaoud, D., Ungerleider, L. G., and Desimone, R. (1990). Pathways for motion analysis: cortical connections of the medial superior temporal and fundus of the superior temporal visual areas in the macaque. J. Comp. Neurol. 296, 462-495. doi: $10.1002 /$ cne.902960311

Briggs, F. (2010). Organizing principles of cortical layer 6. Front. Neural Circuits 4:3. doi: 10.3389/neuro.04.003.2010

Bruce, C., Desimone, R., and Gross, C. G. (1981). Visual properties of neurons in a polysensory area in superior temporal sulcus of the macaque. J. Neurophysiol. 46, 369-384.

Burton, H., and Jones, E. G. (1976). The posterior thalamic region and its cortical projection in New World and Old World monkeys. J. Comp. Neurol. 168, 249-301. doi: 10.1002/cne.901680204

Callaway, E. M. (2002). Cell type specificity of local cortical connections. J. Neurocytol. 31, 231-237. doi: 10.1023/A:1024165824469

Camalier, C. R., D’Angelo, W. R., Sterbing-D'Angelo, S. J., de la Mothe, L. A., and Hackett, T. A. (2012). Neural latencies across auditory cortex of macaque support a dorsal stream supramodal timing advantage in primates. Proc. Natl. Acad. Sci. U.S.A. 109, 18168-18173. doi: 10.1073/pnas.1206387109

Cavada, C., Company, T., Tejedor, J., Cruz-Rizzolo, R. J., and ReinosoSuarez, F. (2000). The anatomical connections of the macaque monkey orbitofrontal cortex. A review. Cereb. Cortex 10, 220-242. doi: 10.1093/cercor/ 10.3.220

Cipolloni, P. B., and Pandya, D. N. (1989). Connectional analysis of the ipsilateral and contralateral afferent neurons of the superior temporal region in the rhesus monkey. J. Comp. Neurol. 281, 567-585. doi: 10.1002/cne.902810407

Cusick, C. G., Seltzer, B., Cola, M., and Griggs, E. (1995). Chemoarchitectonics and corticocortical terminations within the superior temporal sulcus of the rhesus monkey: evidence for subdivisions of superior temporal polysensory cortex. J. Comp. Neurol. 360, 513-535. doi: 10.1002/cne.903600312

de la Mothe, L. A., Blumell, S., Kajikawa, Y., and Hackett, T. A. (2006). Cortical connections of auditory cortex in marmoset monkeys: core and medial belt regions. J. Comp. Neurol. 496, 27-71. doi: 10.1002/cne.20923

de la Mothe, L. A., Blumell, S., Kajikawa, Y., and Hackett, T. A. (2012). Cortical connections of auditory cortex in marmoset monkeys: lateral belt and parabelt regions. Anat. Rec. 295, 800-821. doi: 10.1002/ar.22451

Desimone, R., and Gross, C. G. (1979). Visual areas in the temporal cortex of the macaque. Brain Res. 178, 363-380. doi: 10.1016/0006-8993(79)90699-1

Douglas, R. J., and Martin, K. A. (2007). Mapping the matrix: the ways of neocortex. Neuron 56, 226-238. doi: 10.1016/j.neuron.2007.10.017

Falchier, A., Clavagnier, S., Barone, P., and Kennedy, H. (2002). Anatomical evidence of multimodal integration in primate striate cortex. J. Neurosci. 22, 5749-5759.

Falchier, A., Schroeder, C. E., Hackett, T. A., Lakatos, P., Nascimento-Silva, S., Ulbert, I., et al. (2009). Projection from visual areas V2 and prostriata to caudal auditory cortex in the monkey. Cereb. Cortex 20, 1529-1538. doi: 10.1093/ cercor/bhp213

Feldmeyer, D., Brecht, M., Helmchen, F., Petersen, C. C., Poulet, J. F., Staiger, J. F., et al. (2013). Barrel cortex function. Prog. Neurobiol. 103, 3-27. doi: 10.1016/j.pneurobio.2012.11.002

Felleman, D. J., and Van Essen, D. C. (1991). Distributed hierarchical processing in the primate cerebral cortex. Cereb. Cortex 1, 1-47. doi: 10.1093/cercor/1.1.1

Fitzpatrick, K. A., and Imig, T. J. (1980). Auditory cortico-cortical connections in the owl monkey. J. Comp. Neurol. 192, 589-610. doi: 10.1002/cne.901920314

Galaburda, A. M., and Pandya, D. N. (1983). The intrinsic architectonic and connectional organization of the superior temporal region of the rhesus monkey. J. Comp. Neurol. 221, 169-184. doi: 10.1002/cne.902210206

Gallyas, F. (1979). Silver staining of myelin by means of physical development. Neurol. Res. 1, 203-209.

Geneser-Jensen, F. A., and Blackstad, T. W. (1971). Distribution of acetyl cholinesterase in the hippocampal region of the guinea pig. I. Entorhinal area, parasubiculum, and presubiculum. Z. Zellforsch. Mikrosk. Anat. 114, 460-481. doi: 10.1007/BF00325634

Ghashghaei, H. T., and Barbas, H. (2002). Pathways for emotion: interactions of prefrontal and anterior temporal pathways in the amygdala of the rhesus monkey. Neuroscience 115, 1261-1279. doi: 10.1016/S0306-4522(02)00446-3

Hackett, T. A. (2011). Information flow in the auditory cortical network. Hear. Res. 271, 133-146. doi: 10.1016/j.heares.2010.01.011

Hackett, T. A., and de la Mothe, L. A. (2009). Regional and laminar distribution of the vesicular glutamate transporter, VGluT2, in the macaque monkey auditory cortex. J. Chem. Neuroanat. 38, 106-116. doi: 10.1016/j.jchemneu.2009.05.002

Hackett, T. A., de la Mothe, L. A., Ulbert, I., Karmos, G., Smiley, J., and Schroeder, C. E. (2007). Multisensory convergence in auditory cortex, II. Thalamocortical connections of the caudal superior temporal plane. J. Comp. Neurol. 502, 924-952. doi: 10.1002/cne.21326

Hackett, T. A., Karmos, G., Schroeder, C. E., Ulbert, I., Sterbing-D’Angelo, S. J., D'Angelo, W. R., et al. (2005). Neurosurgical access to cortical areas in the lateral fissure of primates. J. Neurosci. Methods 141, 103-113. doi: 10.1016/j.jneumeth.2004.06.001

Hackett, T. A., Preuss, T. M., and Kaas, J. H. (2001). Architectonic identification of the core region in auditory cortex of macaques, chimpanzees, and humans. J. Comp. Neurol. 441, 197-222. doi: 10.1002/cne.1407

Hackett, T. A., Stepniewska, I., and Kaas, J. H. (1998). Subdivisions of auditory cortex and ipsilateral cortical connections of the parabelt auditory cortex in macaque monkeys. J. Comp. Neurol. 394, 475-495. doi: 10.1002/(SICI)10969861(19980518)394:4<475::AID-CNE6>3.0.CO;2-Z 
Hackett, T. A., Stepniewska, I., and Kaas, J. H. (1999). Prefrontal connections of the parabelt auditory cortex in macaque monkeys. Brain Res. 817, 45-58. doi: 10.1016/S0006-8993(98)01182-2

Hikosaka, K., Iwai, E., Saito, H., and Tanaka, K. (1988). Polysensory properties of neurons in the anterior bank of the caudal superior temporal sulcus of the macaque monkey. J. Neurophysiol. 60, 1615-1637.

Imig, T. J., Ruggero, M. A., Kitzes, L. M., Javel, E., and Brugge, J. F. (1977). Organization of auditory cortex in the owl monkey (Aotus trivirgatus). J. Comp. Neurol. 171, 111-128. doi: 10.1002/cne.901710108

Jones, E. G., and Burton, H. (1976). Areal differences in the laminar distribution of thalamic afferents in cortical fields of the insular, parietal and temporal regions of primates. J. Comp. Neurol. 168, 197-247. doi: 10.1002/cne.901680203

Jones, E. G., Dell'Anna, M. E., Molinari, M., Rausell, E., and Hashikawa, T. (1995). Subdivisions of macaque monkey auditory cortex revealed by calcium-binding protein immunoreactivity. J. Comp. Neurol. 362, 153-170. doi: 10.1002/cne.903620202

Jones, E. G., and Powell, T. P. (1970). An anatomical study of converging sensory pathways within the cerebral cortex of the monkey. Brain 93, 793-820. doi: 10.1093/brain/93.4.793

Kaas, J. H., and Hackett, T. A. (1998). Subdivisions of auditory cortex and levels of processing in primates. Audiol. Neurootol. 3, 73-85. doi: 10.1159/000013783

Kaas, J. H., and Hackett, T. A. (2000). Subdivisions of auditory cortex and processing streams in primates. Proc. Natl. Acad. Sci. U.S.A. 97, 11793-11799. doi: 10.1073/pnas.97.22.11793

Kikuchi, Y., Horwitz, B., and Mishkin, M. (2010). Hierarchical auditory processing directed rostrally along the monkey's supratemporal plane. J. Neurosci. 30, 13021-13030. doi: 10.1523/JNEUROSCI.2267-10.2010

Kosaki, H., Hashikawa, T., He, J., and Jones, E. G. (1997). Tonotopic organization of auditory cortical fields delineated by parvalbumin immunoreactivity in macaque monkeys. J. Comp. Neurol. 386, 304-316. doi: 10.1002/(SICI)10969861(19970922)386:2<304::AID-CNE10>3.0.CO;2-K

Kosmal, A., Malinowska, M., and Kowalska, D. M. (1997). Thalamic and amygdaloid connections of the auditory association cortex of the superior temporal gyrus in rhesus monkey (Macaca mulatta). Acta Neurobiol. Exp. 57, $165-188$.

Kusmierek, P., Ortiz, M., and Rauschecker, J. P. (2012). Sound-identity processing in early areas of the auditory ventral stream in the macaque. J. Neurophysiol. 107, 1123-1141. doi: 10.1152/jn.00793.2011

Kusmierek, P., and Rauschecker, J. P. (2009). Functional specialization of medial auditory belt cortex in the alert rhesus monkey. J. Neurophysiol. 102, 1606-1622. doi: 10.1152/jn.00167.2009

Lakatos, P., Pincze, Z., Fu, K. G., Javitt, D. C., Karmos, G., and Schroeder, C. E. (2005a). Timing of pure tone and noise-evoked responses in macaque auditory cortex. Neuroreport 16, 933-937. doi: 10.1097/00001756-200506210-00011

Lakatos, P., Shah, A. S., Knuth, K. H., Ulbert, I., Karmos, G., and Schroeder, C. E. (2005b). An oscillatory hierarchy controlling neuronal excitability and stimulus processing in the auditory cortex. J. Neurophysiol. 94, 1904-1911. doi: 10.1152/jn.00263.2005

Lavenex, P., Suzuki, W. A., and Amaral, D. G. (2002). Perirhinal and parahippocampal cortices of the macaque monkey: projections to the neocortex. J. Comp. Neurol. 447, 394-420. doi: 10.1002/cne.10243

Leinonen, L., Hyvarinen, J., and Sovijarvi, A. R. (1980). Functional properties of neurons in the temporo-parietal association cortex of awake monkey. Exp. Brain Res. 39, 203-215. doi: 10.1007/BF00237551

Lewis, J. W., and Van Essen, D. C. (2000). Corticocortical connections of visual, sensorimotor, and multimodal processing areas in the parietal lobe of the macaque monkey. J. Comp. Neurol. 428, 112-137. doi: 10.1002/10969861(20001204)428:1\%3C112::AID-CNE8\%3E3.0.CO;2-9

Markov, N. T., Ercsey-Ravasz, M. M., Ribeiro Gomes, A. R., Lamy, C., Magrou, L., Vezoli, J., et al. (2014). A weighted and directed interareal connectivity matrix for macaque cerebral cortex. Cereb. Cortex 24, 17-36. doi: 10.1093/cercor/bhs 270

Markov, N. T., and Kennedy, H. (2013). The importance of being hierarchical. Curr. Opin. Neurobiol. 23, 187-194. doi: 10.1016/j.conb.2012.12.008

Morel, A., Garraghty, P. E., and Kaas, J. H. (1993). Tonotopic organization, architectonic fields, and connections of auditory cortex in macaque monkeys. J. Comp. Neurol. 335, 437-459. doi: 10.1002/cne.903350312

Morel, A., and Kaas, J. H. (1992). Subdivisions and connections of auditory cortex in owl monkeys. J. Comp. Neurol. 318, 27-63. doi: 10.1002/cne.903180104
Padberg, J., Seltzer, B., and Cusick, C. G. (2003). Architectonics and cortical connections of the upper bank of the superior temporal sulcus in the rhesus monkey: an analysis in the tangential plane. J. Comp. Neurol. 467, 418-434. doi: 10.1002/cne. 10932

Pandya, D. N., Hallett, M., and Mukherjee, S. K. (1969). Intra- and interhemispheric connections of the neocortical auditory system in the rhesus monkey. Brain Res. 14, 49-65. doi: 10.1016/0006-8993(69)90030-4

Pandya, D. N., and Kuypers, H. G. (1969). Cortico-cortical connections in the rhesus monkey. Brain Res. 13, 13-36. doi: 10.1016/0006-8993(69)90141-3

Pandya, D. N., and Rosene, D. L. (1993). Laminar termination patterns of thalamic, callosal, and association afferents in the primary auditory area of the rhesus monkey. Exp. Neurol. 119, 220-234. doi: 10.1006/exnr. 1993.1024

Pandya, D. N., and Sanides, F. (1973). Architectonic parcellation of the temporal operculum in rhesus monkey and its projection pattern. Z. Anat. Entwicklungsgesch. 139, 127-161. doi: 10.1007/BF00523634

Pandya, D. N., and Seltzer, B. (1982). Intrinsic connections and architectonics of posterior parietal cortex in the rhesus monkey. J. Comp. Neurol. 204, 196-210. doi: 10.1002/cne.902040208

Petkov, C. I., Kayser, C., Steudel, T., Whittingstall, K., Augath, M., and Logothetis, N. K. (2008). A voice region in the monkey brain. Nat. Neurosci. 11, 367-374. doi: $10.1038 / \mathrm{nn} 2043$

Petrides, M., and Pandya, D. N. (2002). Comparative cytoarchitectonic analysis of the human and the macaque ventrolateral prefrontal cortex and corticocortical connection patterns in the monkey. Eur. J. Neurosci. 16, 291-310. doi: 10.1046/j.1460-9568.2001.02090.x

Rauschecker, J. P. (1998a). Cortical processing of complex sounds. Curr. Opin. Neurobiol. 8, 516-521. doi: 10.1016/S0959-4388(98)80040-8

Rauschecker, J. P. (1998b). Parallel processing in the auditory cortex of primates. Audiol. Neurootol. 3, 86-103. doi: 10.1159/000013784

Rauschecker, J. P., and Tian, B. (2004). Processing of band-passed noise in the lateral auditory belt cortex of the rhesus monkey. J. Neurophysiol. 91, 2578-2589. doi: 10.1152/jn.00834.2003

Rauschecker, J. P., Tian, B., and Hauser, M. (1995). Processing of complex sounds in the macaque nonprimary auditory cortex. Science 268, 111-114. doi: 10.1126/science.7701330

Rauschecker, J. P., Tian, B., Pons, T., and Mishkin, M. (1997). Serial and parallel processing in rhesus monkey auditory cortex. J. Comp. Neurol. 382, 89-103. doi: 10.1002/(SICI)1096-9861(19970526)382:1<89::AID-CNE6>3.0.CO;2-G

Rockland, K. S., and Ojima, H. (2003). Multisensory convergence in calcarine visual areas in macaque monkey. Int. J. Psychophysiol. 50, 19-26. doi: 10.1016/S01678760(03)00121-1

Rockland, K. S., and Pandya, D. N. (1979). Laminar origins and terminations of cortical connections of the occipital lobe in the rhesus monkey. Brain Res. 179, 3-20. doi: 10.1016/0006-8993(79)90485-2

Romanski, L. M., Bates, J. F., and Goldman-Rakic, P. S. (1999a). Auditory belt and parabelt projections to the prefrontal cortex in the rhesus monkey. J. Comp. Neurol. 403, 141-157. doi: 10.1002/(SICI)1096-9861(19990111)403:2<141::AID-CNE1>3.0.CO;2-V

Romanski, L. M., Tian, B., Fritz, J., Mishkin, M., Goldman-Rakic, P. S., and Rauschecker, J. P. (1999b). Dual streams of auditory afferents target multiple domains in the primate prefrontal cortex. Nat. Neurosci. 2, 1131-1136. doi: $10.1038 / 16056$

Saleem, K. S., Kondo, H., and Price, J. L. (2008). Complementary circuits connecting the orbital and medial prefrontal networks with the temporal, insular, and opercular cortex in the macaque monkey. J. Comp. Neurol. 506, 659-693. doi: 10.1002/cne. 21577

Saleem, K. S., and Logothetis, N. K. (2012). A Combined MRI and Histology Atlas of the Rhesus Monkey Brain in Stereotaxic Coordinates, 2nd Edn. with Horizontal, Coronal and Sagittal series. San Diego: Elsevier; Academic Press.

Saleem, K. S., Miller, B., and Price, J. L. (2013). Subdivisions and connectional networks of the lateral prefrontal cortex in the macaque monkey. J. Comp. Neurol. 522, 1641-1690. doi: 10.1002/cne.23498

Saleem, K. S., Suzuki, W., Tanaka, K., and Hashikawa, T. (2000). Connections between anterior inferotemporal cortex and superior temporal sulcus regions in the macaque monkey. J. Neurosci. 20, 5083-5101.

Schroeder, C. E., and Foxe, J. J. (2002). The timing and laminar profile of converging inputs to multisensory areas of the macaque neocortex. Brain Res. Cogn. Brain Res. 14, 187-198. doi: 10.1016/S0926-6410(02)00073-3 
Scott, B. H., Malone, B. J., and Semple, M. N. (2011). Transformation of temporal processing across auditory cortex of awake macaques. J. Neurophysiol. 105, 712-730. doi: 10.1152/jn.01120.2009

Seltzer, B., Cola, M. G., Gutierrez, C., Massee, M., Weldon, C., and Cusick, C. G. (1996). Overlapping and nonoverlapping cortical projections to cortex of the superior temporal sulcus in the rhesus monkey: double anterograde tracer studies. J. Comp. Neurol. 370, 173-190. doi: 10.1002/(SICI)10969861(19960624)370:2<173::AID-CNE4>3.0.CO;2-\#

Seltzer, B., and Pandya, D. N. (1978). Afferent cortical connections and architectonics of the superior temporal sulcus and surrounding cortex in the rhesus monkey. Brain Res. 149, 1-24. doi: 10.1016/0006-8993(78)90584-X

Seltzer, B., and Pandya, D. N. (1989a). Frontal lobe connections of the superior temporal sulcus in the rhesus monkey. J. Comp. Neurol. 281, 97-113. doi: 10.1002/ cne. 902810108

Seltzer, B., and Pandya, D. N. (1989b). Intrinsic connections and architectonics of the superior temporal sulcus in the rhesus monkey. J. Comp. Neurol. 290, 451-471. doi: 10.1002/cne.902900402

Seltzer, B., and Pandya, D. N. (1994). Parietal, temporal, and occipital projections to cortex of the superior temporal sulcus in the rhesus monkey: a retrograde tracer study. J. Comp. Neurol. 343, 445-463. doi: 10.1002/cne.903430308

Smiley, J. F., Hackett, T. A., Ulbert, I., Karmas, G., Lakatos, P., Javitt, D. C., et al. (2007). Multisensory convergence in auditory cortex, I. Cortical connections of the caudal superior temporal plane in macaque monkeys. J. Comp. Neurol. 502, 894-923. doi: 10.1002/cne.21325

Thomson, A. M., and Bannister, A. P. (2003). Interlaminar connections in the neocortex. Cereb. Cortex 13, 5-14. doi: 10.1093/cercor/13.1.5

Tranel, D., Brady, D. R., Van Hoesen, G. W., and Damasio, A. R. (1988). Parahippocampal projections to posterior auditory association cortex (area Tpt) in Old-World monkeys. Exp. Brain Res. 70, 406-416. doi: 10.1007/BF00248365
Ungerleider, L. G., and Desimone, R. (1986). Projections to the superior temporal sulcus from the central and peripheral field representations of V1 and V2. J. Comp. Neurol. 248, 147-163. doi: 10.1002/cne.902 480202

Wong-Riley, M. (1979). Changes in the visual system of monocularly sutured or enucleated cats demonstrable with cytochrome oxidase histochemistry. Brain Res. 171, 11-28. doi: 10.1016/0006-8993(79)90728-5

Yukie, M. (2002). Connections between the amygdala and auditory cortical areas in the macaque monkey. Neurosci. Res. 42, 219-229. doi: 10.1016/S01680102(01)00325-X

Conflict of Interest Statement: The authors declare that the research was conducted in the absence of any commercial or financial relationships that could be construed as a potential conflict of interest.

Received: 24 January 2014; accepted: 25 March 2014; published online: 22 April 2014. Citation: Hackett TA, de la Mothe LA, Camalier CR, Falchier A, Lakatos P, Kajikawa $Y$ and Schroeder CE (2014) Feedforward and feedback projections of caudal belt and parabelt areas of auditory cortex: refining the hierarchical model. Front. Neurosci. 8:72. doi: 10.3389/fnins.2014.00072

This article was submitted to Auditory Cognitive Neuroscience, a section of the journal Frontiers in Neuroscience.

Copyright (C) 2014 Hackett, de la Mothe, Camalier, Falchier, Lakatos, Kajikawa and Schroeder. This is an open-access article distributed under the terms of the Creative Commons Attribution License (CC BY). The use, distribution or reproduction in other forums is permitted, provided the original author(s) or licensor are credited and that the original publication in this journal is cited, in accordance with accepted academic practice. No use, distribution or reproduction is permitted which does not comply with these terms. 